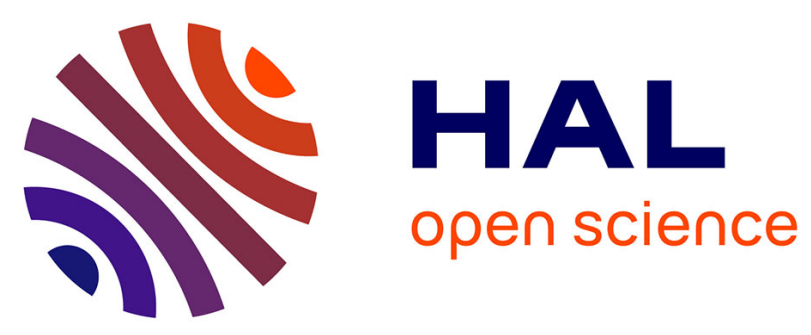

\title{
La provenance des barres de fer romaines des Saintes-Maries-de-la-Mer (Bouches-du-Rhône) : étude archéologique et archéométrique
}

Marie-Pierre Coustures, Christian Rico, Didier Béziat, David Djaoui, Luc Long, Claude Domergue, Francis Tollon

\section{To cite this version:}

Marie-Pierre Coustures, Christian Rico, Didier Béziat, David Djaoui, Luc Long, et al.. La provenance des barres de fer romaines des Saintes-Maries-de-la-Mer (Bouches-du-Rhône): étude archéologique et archéométrique. Gallia - Archéologie de la France antique, 2006, 63, pp.243-261. 10.3406/galia.2006.3297 . hal-01911027

\section{HAL Id: hal-01911027 \\ https://hal.science/hal-01911027}

Submitted on 8 Jan 2020

HAL is a multi-disciplinary open access archive for the deposit and dissemination of scientific research documents, whether they are published or not. The documents may come from teaching and research institutions in France or abroad, or from public or private research centers.
L'archive ouverte pluridisciplinaire HAL, est destinée au dépôt et à la diffusion de documents scientifiques de niveau recherche, publiés ou non, émanant des établissements d'enseignement et de recherche français ou étrangers, des laboratoires publics ou privés.

\section{(ㅇ)(1) $\$$}

Distributed under a Creative Commons Attribution - NonCommercial - NoDerivatives 44.0 


\title{
LA PROVENANCE DES BARRES DE FER ROMAINES DES SAINTES-MARIES- DE-LA-MER (BOUCHES-DU-RHÔNE)
}

\section{Étude archéologique et archéométrique}

\author{
Marie-Pierre Coustures*, Christian RICO*, Didier BÉZIAT ${ }^{* *}$, David DJAOuI ${ }^{* * *}$, \\ Luc LONG***, Claude DOMERGUE*, Francis TOLlON**
}

\begin{abstract}
Mots-clés. Montagne Noire, épaves romaines, barres de fer, épigraphie, inclusions de scories, analyses chimiques.
Résumé. Cet article a deux objectifs: d'une part, faire connaître l'état actuel des découvertes d'épaves romaines chargées de fer devant les Saintes-Maries-de-la-Mer (une nouvelle typologie, ouverte, raisonnée et qui intègre l'ancienne est proposée) et, d'autre part, par le biais des analyses chimiques (éléments en trace) d'inclusions de scorie, tester l'hypothèse de provenance du centre sidérurgique des Martys.

Si l'étude archéométrique a permis de montrer qu'une partie de ce fer pouvait effectivement provenir de la Montagne Noire, elle a également mis en évidence l'existence d'au moins deux autres régions de production, encore indéterminées, pour l'origine de ces cargaisons. Faut-il chercher ces autres centres de production dans une zone géographiquement proche, comme semblent le suggérer l'étude onomastique et la mise en évidence de points communs dans le mode d'agencement des estampilles - ce qui orienterait plutôt les recherches vers les Corbières ou les Pyrénées - ou bien plus loin dans le monde romain? La multiplication de ce genre d'études ne manquera certainement pas de répondre aux nouvelles questions qui se posent sur la provenance des cargaisons de fer des épaves des Saintes-Maries-de-la-Mer.
\end{abstract}

Key-words. Montagne Noire, Roman wrecks, iron bars, epigraphy, slag inclusions, chemical analyses.

Abstract. This paper has two aims: first, to set out the present state of discoveries of Roman wrecks loaded with iron in front of les Saintes-Maries-de-la-Mer ( a new typology, open and reasoned, is proposed, which integrates the former one) and, on the other hand, by the means of chemical analyses (elements in trace) of the entrapped slag inclusions, to express the hypothesis of origin of the iron-making centre of Les Martys.

The archaeometrical study showed that part of the iron could actually come from la Montagne Noire but it also demonstrates that there were at least two other origins, from iron-making centres still unknown. Are they situated in a zone near Les Martys, what the onomastic study and the common points observed in the way to mark the bars seem to indicate? In that case one of these areas could be situated in the Corbières mountain or in the Pyrenean range or farther on in the Roman world. The increasing number of this kind of studies should give an answer to these new questions about the origin of the cargoes of the wrecks of les Saintes-Maries-de-la-Mer.

Translation: Isabelle FAUDUET

\footnotetext{
* UMR 5608 du CNRS, Unité toulousaine d'archéologie et d'histoire (UTAH), Maison de la recherche, Université de Toulouse-Le Mirail, 5 allées Antonio-Machado, F-31058 Toulouse Cedex 9. Courriels: maripicou@laposte.net; claude.domergue@wanadoo.fr; chrico@club-internet.fr

** UMR 5563 du CNRS, Laboratoire des mécanismes et transferts en géologie (LMTG), Université Paul-Sabatier, 14 avenue Édouard-Belin, F-31400 Toulouse. Courriel: dbeziat@lmtg.obs-mip.fr

**** Musée de l’Arles et de la Provence antiques, Presqu'île du Cirque Romain, BP 205, F-13635 Arles Cedex.

**** Département des recherches archéologiques subaquatiques et sous-marines (DRASSM), Fort Saint-Jean, F-13235 Marseille Cedex 2. Courriel: luc.long@culture.gouv.fr
} 
Schlagwörter. Montagne Noire, römische Schiffswracks, Eisenbarren, Epigraphik, Schlackeneinschlüsse, chemische Analyse, Herkunftsfrage.

Zusammenfassung. Dieser Beitrag verfolgt zwei Ziele. Zum einen soll der aktuelle Kenntnisstand der mit Eisen beladenen römischen Schiffswracks bekanntgemacht werden, die vor Saintes-Maries-de-la-Mer entdeckt worden sind. Dabei wird eine neue, erweiterte und systematische aufgebaute Typologie vorgeschlagen, die allerdings die bestehende weiter gelten läßt. Zum anderen wurde anhand der Ergebnisse der chemischen Analyse der Schlackeneinschlüsse (Spurenelemente) die Hypothese einer Herkunft aus dem Eisenproduktionszentrum von Les Martys verfolgt.

Die archäometrische Untersuchung konnte zeigen, daß ein Teil des fraglichen Eisens tatsächlich aus der Montagne Noire stammen kann. Im gleichen Zuge ist aber auch klar geworden, daß für die Herkunft der Schiffsladungen von mindestens zwei weiteren, bisher nicht identifizierten Produktionsregionen auszugehen ist. Muß man diese anderen Produktionszentren in einem engeren geographischen Umfeld suchen, wie es die namenskundliche Untersuchung und die deutlich gewordenen Gemeinsamkeiten hinsichtlich der Anbringung der Marken nahezulegen scheinen (was die Suche eher in Richtung der Corbières oder der Pyrenäen lenken würde), oder eher weiter entfernt in der römischen Welt? Bestimmt werden vermehrte Untersuchungen dieser Art auf die Fragen Antwort geben, die sich zur Herkunft der Eisenladungen der Wracks von Saintes-Maries-de-la-Mer gegenwärtig stellen.

Übersetzung: Stefan WIRTH

Le nombre d'épaves antiques que le Département des recherches archéologiques subaquatiques et sous-marines (DRASSM) a répertoriées en un peu plus de vingt ans au large des Saintes-Maries-de-la Mer dépasse aujourd'hui la trentaine. Dix sont remarquables par leur chargement de barres de fer: aux sept épaves connues en 2000 (SM2, SM3, SM6, SM8, SM9, SM10, SM11: Long, 1997; Long et al., 2002), il faut en effet ajouter trois nouvelles découvertes, faites respectivement en 2001 (SM23), en 2002 (SM24) (Long, 2002) et en 2005 (SM25). Les expertises effectuées sur la plupart de ces épaves ont montré que leurs cargaisons de fer représentaient des dizaines de tonnes et témoignaient d'un important commerce de ce métal dans la Méditerranée occidentale au I $^{\text {er }}$ s. av. J.-C. et au I ${ }^{\text {er }}$ s. apr. J.-C. ${ }^{1}$.

La position de ces épaves est éloquente; elles sont situées face à l'embouchure d'un ancien bras du Rhône (fig. 1). Le naufrage des navires est attribué à l'existence d'une barre constituée par des bancs de sable difficiles à franchir pour des bateaux de faible tirant d'eau (Long, 1995, p. 42; Long, Sintès, 2003, p. 189-197). Ces bateaux devaient vouloir s'engager dans le Rhône et le remonter ${ }^{2}$. Ces remarques et la chronologie des épaves ont fait penser que le fer qu'elles

1. L'expertise de SM25 n'a livré aucun matériel significatif permettant de dater cette épave. Néanmoins, la très grande similitude des barres de fer qu'elle renferme avec celles des autres épaves connues (SM2 notamment) milite en faveur d'une datation à l'époque romaine.

2. Une découverte toute récente (sept. 2005) dans le Rhône, dans la zone portuaire d'Arles (en cours d'étude par le DRASSM), en apporte une première illustration. Il s'agit d'une barre de fer en tous points identique à celles des Saintes-Maries, et assurément antique en raison des deux timbres rectangulaires dont elle est frappée. L'interprétation des inscriptions, Acirina pour l'une, Turonicu(s) pour l'autre (fig. 5, $\mathrm{n}^{\text {os }} 1$ et 2), qui renvoient, semble-t-il, au monde gaulois, pose encore problème. renfermaient pouvait être destiné, au moins en partie, aux armées du Rhin (Long et al., 2002, p. 183) ${ }^{3}$, qui avaient besoin d'un approvisionnement massif et régulier.

L'origine de ce fer est une autre question. Dans l'hypothèse que l'on vient de faire sur l'itinéraire suivi par les bateaux et sur la destination du fer, on ne peut chercher la ou les région(s) d'origine de ce métal que dans l'ouest du monde romain. À la période considérée, nous ne connaissons guère que deux zones importantes alors en activité: le massif du Canigou et la Montagne Noire ${ }^{4}$ (fig. 2).

3. La même interprétation avait été proposée pour une épave chargée de lingots de plomb ( $S M 1)$, découverte également dans les parages (Long, Domergue, 1995, p. 835) ; ces lingots, d'abord attribués à l'Espagne (id., ibid., p. 806 et 834) semblaient provenir des mines des Cévennes (Trincherini et al., 2001). Mais de récentes publications ont proposé une autre interprétation: ces lingots de plomb seraient originaires des mines de Germanie (Rothenhöfer, 2003a et 2003b). La question est complexe et met en jeu des arguments d'ordre archéologique, historique et épigraphique auxquels s'ajoute le problème des signatures isotopiques; elle est pour l'heure en suspens. Si cette origine était finalement démontrée, cela signifierait que les épaves romaines présentes devant les Saintes-Maries-de-la-Mer ne sont pas toutes celles de navires qui cherchaient à remonter le Rhône, mais que certains de ces bateaux venaient au contraire de le descendre pour transporter leur chargement vers le monde méditerranéen. Mais cela n'impliquerait pas que toutes ces épaves sont celles de navires qui venaient de descendre le Rhône. Nous continuons donc à raisonner sur l'hypothèse de navires chargés de fer qui se préparaient à remonter le cours du fleuve.

4. Une activité sidérurgique est également attestée dans les Corbières à cette période, mais elle paraît assez dispersée et ne présente pas de vestiges (ferriers) aussi imposants que ceux du Canigou et surtout de la Montagne Noire (Rancoule, 1975; Pauc, Pauc, 1998). En Espagne d'autre part, si la Sierra Nevada (Grenade) et la Sierra Menera (Teruel) ont produit du fer en assez grande quantité au second âge du Fer (fig. 2), cette production semble s'être arrêtée, ou à tout le moins avoir décliné à l'époque qui nous intéresse (Long et al., 2002, p. 185-186). 


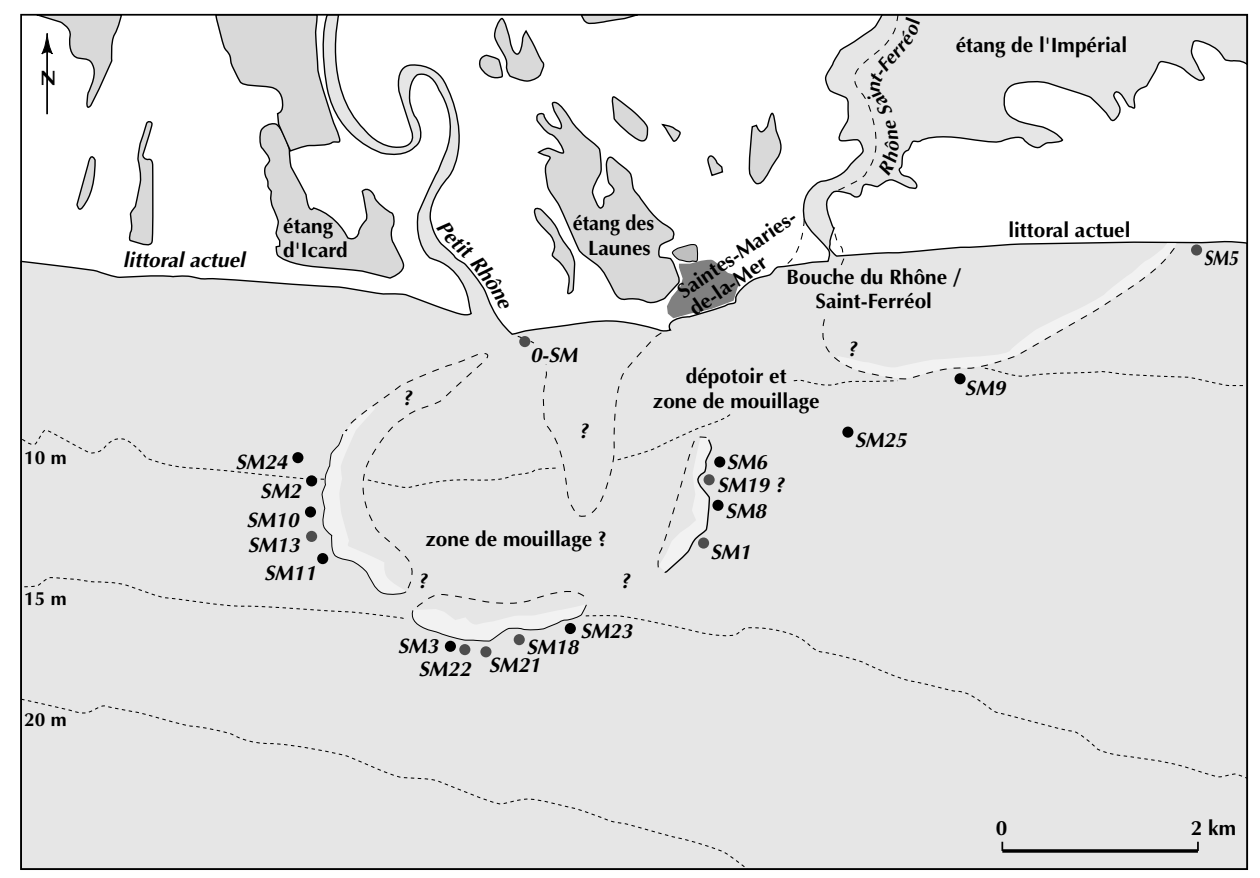

Fig. 1 - Essai de restitution du paléorivage devant l'embouchure du Rhône à Saint-Ferréol, d'après la position des épaves antiques (d'après Long, Sintès, 2003, p. 193). En gras, les épaves chargées de barres de fer (DAO C. Rico, UTAH).

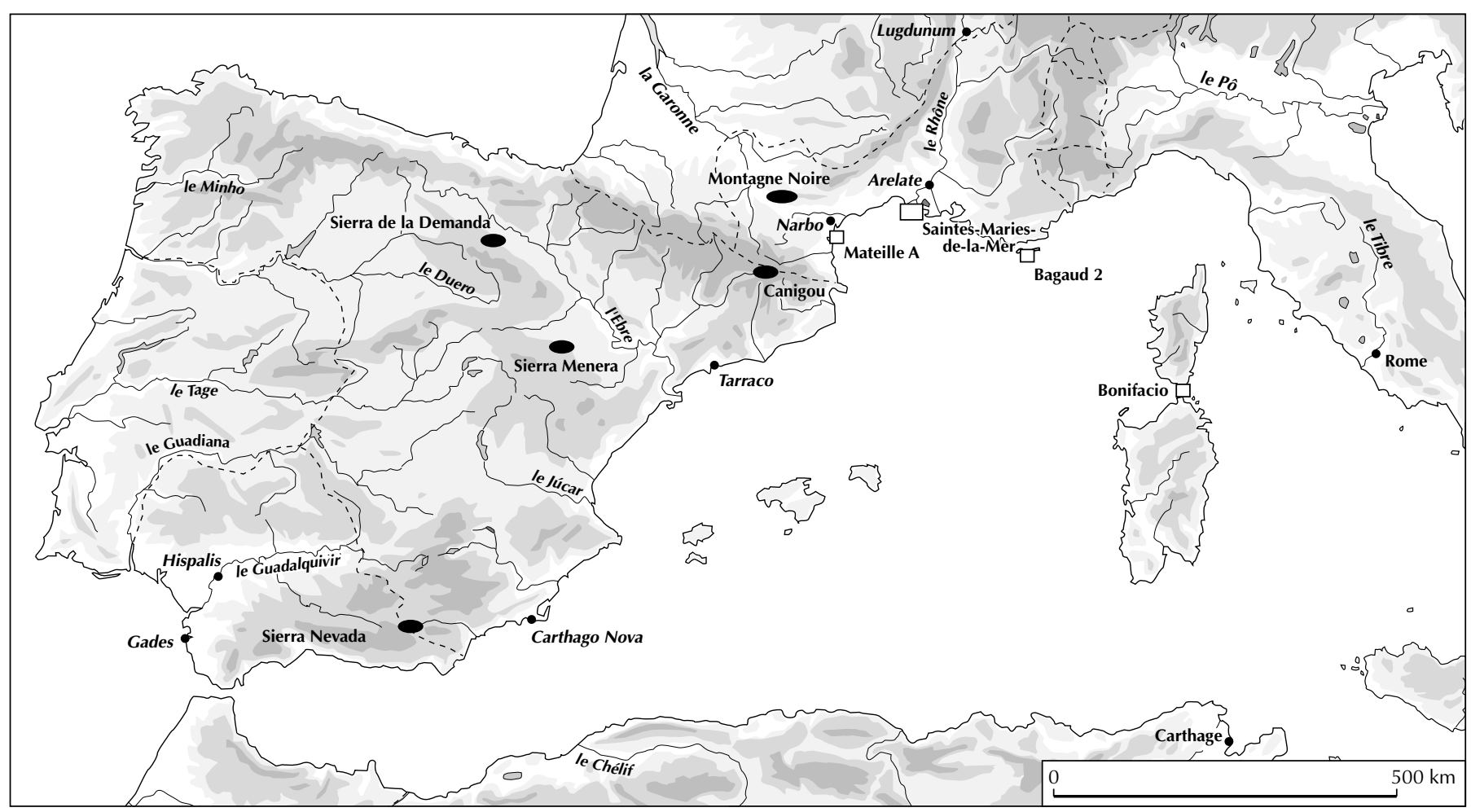

Fig. 2 - Régions et sites mentionnés dans l'article concernant la production et le commerce du fer dans le sud de la Gaule et en péninsule Ibérique au Irr s. av. J.-C. et au I'r s. apr. J.-C. (DAO C. Rico, UTAH). 
Fig. 3 - Concrétion de l'épave SM24 (2003) (cliché C. Durand, Centre Camille-Jullian).

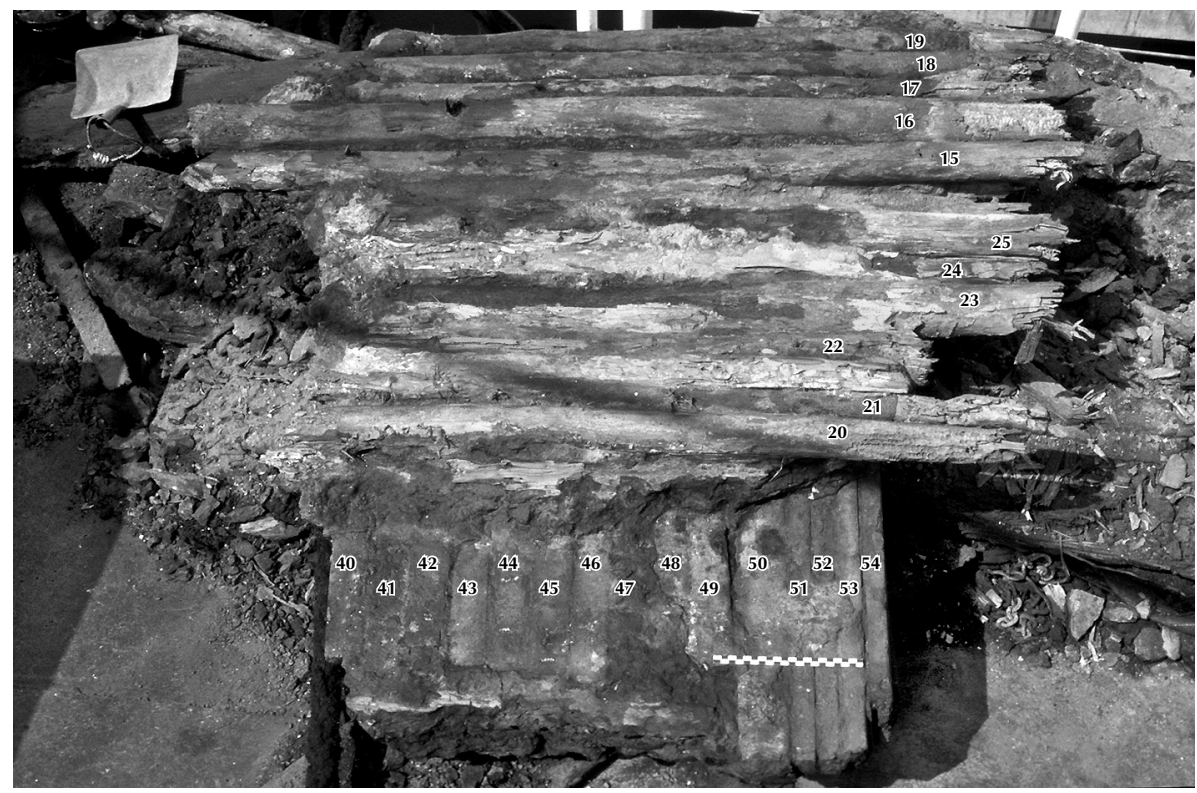

Dans le premier secteur, des prospections ont montré l'existence de tas de scories antiques, dont certains sont assez volumineux (Barrouillet et al., 1991), mais il n'y a pas eu de recherches approfondies et, en tout cas, le Canigou ne saurait rivaliser avec la Montagne Noire ni par le nombre de ferriers, ni par la quantité globale de scories qu'ils renferment. Ajoutons que, dans cette dernière région, le site de référence des Martys est fouillé depuis 1972, que des découvertes spectaculaires y ont été effectuées (en particulier des batteries de bas fourneaux du I ${ }^{\mathrm{er}}$ s. av. J.-C. : Domergue dir., 1993) ; que les minerais, scories et autres produits ont été étudiés et analysés (Jarrier, 1993; Jarrier et al., 1995, 1996; Andrieux et al., 1997), que de nombreux échantillons sont disponibles, que la production de fer globale du site même des Martys a été évaluée à 37000 t (Decombeix et al., 1998) et celle de la région environnante (bassin supérieur de la Dure, y compris Les Martys) à quelque $80000 \mathrm{t}$ (Decombeix et al., 2000), bref qu'il y avait là un fort potentiel d'exportation. On ne s'étonnera donc pas que la Montagne Noire, et en premier lieu Les Martys, se soit imposée à nous comme premier secteur à envisager comme zone de production possible des cargaisons de fer des Saintes-Maries-de-la-Mer; d'où l'intervention des minéralogistes et des métallurgistes pour tenter de résoudre ce problème en comparant, comme on le verra ci-dessous, les matériaux issus de la Montagne Noire, et principalement des Martys (minerai, scories, métal: Coustures et al., 2003), avec le fer des barres des épaves des Saintes-Maries-de-la-Mer.
Mais, tout d'abord, étant donné les nouvelles découvertes qui se sont produites depuis notre dernière synthèse (Long et al., 2002), nous présentons un état de la question, du point de vue tant de l'archéologie que de l'épigraphie.

\section{DESCRIPTION DES BARRES DE FER ET DE LEURS ESTAMPILLES}

Les expertises réalisées depuis 1991 sur les épaves de Camargue ont permis, à chaque fois, de remonter une ou plusieurs concrétions métalliques, dont le débitage, réalisé sur la plage arrière de l'Archéonaute, a donné au total plusieurs centaines de barres de fer. La grande variété de ces dernières a conduit à l'élaboration d'une typologie en 1997 (Long, 1997, reprise dans Long et al., 2002), que la dernière expertise effectuée en 2003 sur l'épave SM24 (fig. 3) est venue compléter (fig. 4). On supposait que ces barres devaient porter des marques de production ou de commerce et nos hypothèses se sont avérées exactes. Le corpus des marques épigraphiques sur barres de fer s'est considérablement enrichi avec les découvertes de Camargue: pas moins de 18 nouvelles marques ont été à ce jour identifiées, qui viennent s'ajouter aux trois connues jusqu'alors ${ }^{5}$.

5. Timbres FERRO à Ben Afelí (Espagne), SATVRNINI à Bonifacio, HAEDVI à Palavas-les-Flots (Long et al., 2002, p. 179 et n. 16-18). Aux Saintes-Maries-de-la-Mer, plusieurs des barres remontées de 

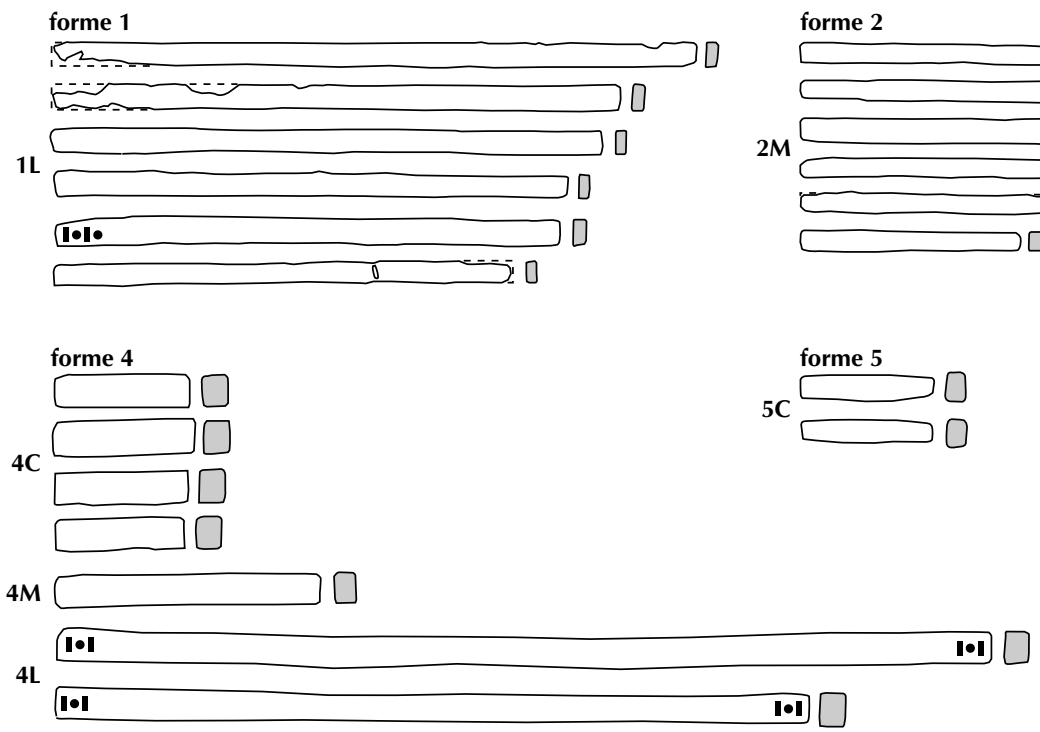
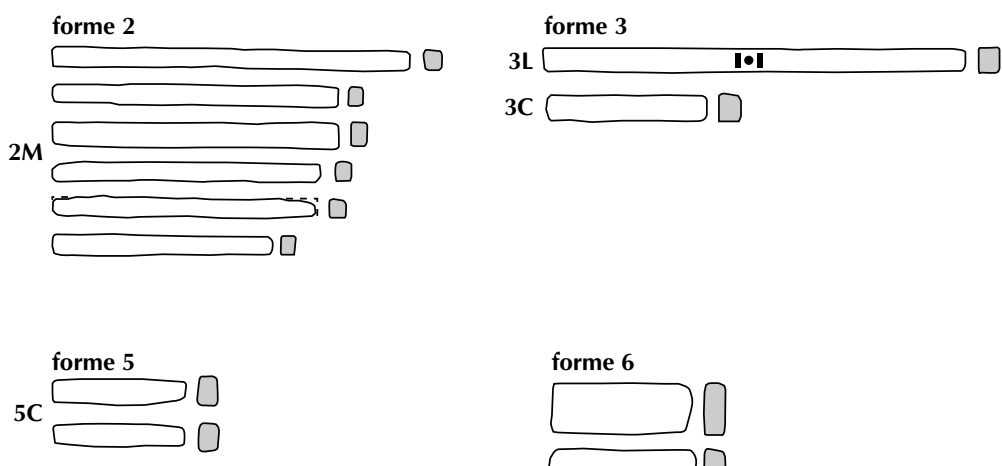

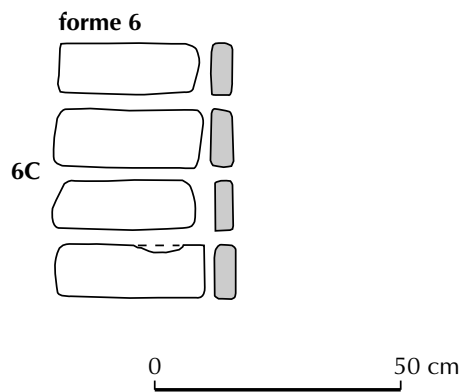

Fig. 4 - Typologie des barres de fer antiques des épaves des Saintes-Maries-de-la-Mer (DAO L. Long, DRASSM et C. Rico, UTAH).

\section{TYPOLOGIE DES BARRES DE FER DES ÉPAVES DES SAINTES-MARIES-DE-LA-MER}

La première impression est celle d'un jeu de formes assez désordonné et peu caractérisé. Nous avons cependant pu définir un certain nombre de catégories, en tenant compte de la morphologie générale des barres, de leur longueur et de leur section. Un premier classement avait abouti à une typologie comprenant six formes, qui a fonctionné depuis 1997 sur un très grand ensemble de masses et de barres de fer recensées dans six épaves: SM2, SM3, SM6, SM8, SM9, $S M 10$. Cette première typologie vient d'être quelque peu remaniée à l'issue des dernières recherches en mer menées sur l'épave SM24 (Long, 2002). Dans cette dernière, une cinquantaine de barres ont été prélevées, parmi lesquelles se distinguent de très longs exemplaires (jusqu'à 1,90 m), trapus, de section à peu près carrée et d'un poids très élevé (jusqu'à 33 kg), que nous avons d'abord considérés comme des formes nouvelles. En ce qui concerne leur section, ces barres correspondent en réalité à des exemplaires déjà connus, mais jusque-là de petite taille (formes 3 et 4). Cette constatation a permis de vérifier que les formes

SM24 en 2003 portent un timbre jusqu'alors inédit: MANI. L'état de corrosion des estampilles ne permet pas de s'assurer que toutes les barres de l'épave étaient frappées de cette seule estampille. Par ailleurs, les nouveaux prélèvements effectués sur SM9 en août 2005 ont apporté leur lot de marques, la plupart déjà connues; quelques autres, bien qu'entièrement corrodées, semblent inédites: nouveau système d'estampillage 6 (tabl. II). antérieurement définies pouvaient varier considérablement en longueur. D'où la nécessité d'élaborer une nouvelle typologie, qui soit ouverte et dans laquelle les formes se définissent par leur section quasi constante, et se déclinent en trois variantes en fonction de leur taille: courte, moyenne et longue. Les formats courts sont généralement inférieurs à $40 \mathrm{~cm}$, les moyens sont compris entre 40 et $75 \mathrm{~cm}$, tandis qu'au-delà, les formats longs peuvent approcher et même dépasser $2 \mathrm{~m}$. Une telle typologie comprend des variantes qui ne sont pas encore attestées à ce jour dans les épaves des Saintes-Maries-de-la-Mer, mais pourraient bien apparaître dans un chargement, lors d'une future campagne de fouille. Le nouveau classement s'établit donc comme suit (tabl. I et fig. 4) ${ }^{6}$ :

La forme 1 se distingue par son profil aplati. En général, la largeur et la hauteur sont proches respectivement de $4 \mathrm{~cm}$ $( \pm 1 \mathrm{~cm})$ et $2 \mathrm{~cm}( \pm 0,5 \mathrm{~cm})$. Le rapport largeur/hauteur est strictement supérieur à $1,8 \mathrm{~cm}$. Jusqu'à présent cette forme, identifiée sur cinq épaves, n'existe pas dans la version courte.

La forme 2, attestée elle aussi dans plusieurs épaves, correspond à des barreaux minces, dont la section relativement fine est carrée ou proche du carré. Les largeur et hauteur moyennes (respectivement $3,5 \mathrm{~cm}$ et $2,5 \mathrm{~cm}$ ) peuvent varier de $\pm 0,5 \mathrm{~cm}$. Dans ce module, proche de

6. Le tableau utilise les données consignées sur les cahiers de fouilles et réunies, épave par épave, dans les tableaux du mémoire de DEA de D. Djaoui (2003). 
Tabl. I - Les caractères typologiques des barres de fer des épaves antiques de Camargue. Dans la colonne 3, la section (largeur x hauteur) permet d'identifier la forme, la longueur de définir la variante (longue, moyenne, courte).

\begin{tabular}{|c|c|c|c|c|c|}
\hline Forme & Désignation & \begin{tabular}{|c|} 
Dimensions \\
largeur $\mathrm{x}$ hauteur $(\mathrm{en} \mathrm{cm})$
\end{tabular} & Constante & $\begin{array}{l}\text { Poids } \\
\text { (en kg) }\end{array}$ & Épave \\
\hline 1 & barre rectiligne, aplatie & $4 \pm 1,3 \times 2 \pm 0,5$ & largeur/hauteur $\geq 1,8$ & - & - \\
\hline $1 C$ & forme courte & - & longueur $<40$ & - & forme non attestée \\
\hline $1 M$ & forme moyenne & longueur 54 à 61 & longueur $>40<72$ & 2,5 à 4,2 & SM3, SM9 \\
\hline 1L & forme longue & longueur 74 à 138,5 & longueur $>72$ & 3,9 à 11,7 & SM2, SM9, SM10, SM24 \\
\hline 2 & $\begin{array}{l}\text { barre et barreau minces, section } \\
\text { carrée ou proche du carré }\end{array}$ & $3,5 \pm 0,5 \times 2,5 \pm 0,5$ & $\begin{array}{c}\text { si largeur } \geq 3,5 \\
\text { hauteur }<3\end{array}$ & - & - \\
\hline $2 \mathrm{C}$ & forme courte & - & longueur $<40$ & - & forme non attestée \\
\hline $2 M$ & forme moyenne & longueur 40 à 71 & longueur $>40<72$ & 1,5 à 5 & SM6, SM8, SM9, SM23, SM25 \\
\hline $2 \mathbf{L}$ & forme longue & - & longueur $>72$ & - & forme non attestée \\
\hline 3 & $\begin{array}{l}\text { barre et barreau } \\
\text { «intermédiaires» }\end{array}$ & $4 \pm 0,5 \times 3,5 \pm 0,5$ & $\begin{array}{c}\text { si largeur }=4,5 \\
\text { hauteur }<3,5\end{array}$ & - & - \\
\hline $3 C$ & forme courte & longueur 30 à 38 & longueur $<40$ & - & SM6, SM8 \\
\hline $3 M$ & forme moyenne & - & longueur $>40<72$ & - & forme non attestée \\
\hline $3 \mathrm{~L}$ & forme longue & longueur 85 & longueur $>72$ & - & SM24 \\
\hline 4 & $\begin{array}{c}\text { barre parallélépipédique } \\
\text { massive }\end{array}$ & $6 \pm 1,8 \times 5 \pm 1,5$ & largeur/hauteur $<1,8$ & - & - \\
\hline $4 C$ & forme courte & longueur 20,8 à 29,9 & longueur $<40$ & 2 à 7 & SM2, SM6, SM9, SM10, SM24 \\
\hline $4 M$ & forme moyenne & - & longueur $>40<72$ & - & forme non attestée \\
\hline $4 \mathrm{~L}$ & forme longue & longueur 76 à 191 & longueur $>72$ & 22 à 33 & SM24 \\
\hline 5 & barre légèrement renflée & $\begin{array}{c}\text { proche de la forme } 3 \\
5 \pm 0,5 \times 3,5\end{array}$ & $\begin{array}{l}\text { largeur max. " } 4 / 3 \\
\text { des extrémités }\end{array}$ & - & - \\
\hline $5 C$ & forme courte & longueur 26 à 31 & longueur $<40$ & 2,9 à 3 & SM6 \\
\hline $5 M$ & forme moyenne & - & longueur $>40<72$ & - & forme non attestée \\
\hline $5 \mathrm{~L}$ & forme longue & - & longueur $>72$ & - & forme non attestée \\
\hline 6 & $\begin{array}{c}\text { plaque aux extrémités } \\
\text { parfois arrondies }\end{array}$ & $10 \pm 0,5 \times 3,7 \pm 0,7$ & - & - & - \\
\hline $6 C$ & forme courte & longueur 27 à 33 & longueur $<40$ & 4,4 à 8,2 & SM6, Mateille A \\
\hline $6 M$ & forme moyenne & - & longueur $>40<72$ & - & forme non attestée \\
\hline $6 \mathrm{~L}$ & forme longue & - & longueur $>72$ & - & forme non attestée \\
\hline
\end{tabular}

la forme 3, lorsque la largeur est supérieure à $3,5 \mathrm{~cm}$, la hauteur est strictement inférieure à $3 \mathrm{~cm}^{7}$. La variante longue de la forme 2 n'est pas encore attestée.

La forme 3 fut d'abord recensée dans sa version courte. Il s'agit d'un pain parallélépipédique, de section pseudocarrée, intermédiaire entre les formes 2 et 4 . Ses dimensions moyennes sont de $4 \mathrm{~cm}$ de large pour $3,5 \mathrm{~cm}$ de haut $( \pm 0,5 \mathrm{~cm})$. L'un des paramètres d'identification est le suivant: lorsque la largeur égale $4,5 \mathrm{~cm}$, la hauteur est alors strictement inférieure à $3,5 \mathrm{~cm}$. Attesté sur deux gisements

7. Ce type de barres fait aussi partie du chargement de l'épave Bagaud 2 dans les Îles d'Hyères, de chronologie plus ancienne que les épaves des Saintes-Maries-de-la-Mer (fin du $\mathrm{II}^{\mathrm{e}}$ s. av. J.-C./début du I ${ }^{\text {er }}$ s. apr. J.-C.). On y trouve aussi quelques barres apparentées à la forme 4C des Saintes-Maries-de-la-Mer (Long, 1985, p. 94 sq.; Long, Valente, 2003, p. 149-150). sous-marins, le format court était jusqu'à présent le plus courant, à l'exception de quelques formes moyennes dans SM6 et d'une forme longue dans SM10, mais l'épave SM24 vient tout récemment de livrer plusieurs exemplaires de très grandes dimensions. L'un d'eux avait d'abord été pris pour une forme 2 (Long, 2002, p. 53).

La forme 4 est illustrée par des pains et des barres parallélépipédiques très massives, qui se distinguent par là de la forme précédente. Sur ce modèle particulier, la largeur et la hauteur, qui atteignent respectivement en moyenne $6 \mathrm{~cm}$ et $5 \mathrm{~cm}$, peuvent accuser des variations de $\pm 1,5 \mathrm{~cm}$. Jusqu'à présent, les barres de forme 4C (fig. 6) sont toujours plus courtes que leurs homologues de forme 3. Pour leur part, les grandes barres jusque-là atypiques, récemment mises au jour dans l'épave SM24 (1,91 m de long pour $33 \mathrm{~kg}$ ), 
méritaient pour certains des auteurs d'être classées dans une nouvelle forme. Mais, d'une part, il n'est pas souhaitable de multiplier à l'infini le nombre des formes, d'autre part, ces grandes barres massives illustrent pleinement la variante longue de la forme 4 . Le format moyen, quant à lui, n'est attesté qu'à un seul exemplaire dans l'épave SM6, mais, compte tenu de la variété du chargement de l'épave SM24, il ne serait pas surprenant d'en trouver d'autres ultérieurement.

La forme 5 désigne visiblement un pain d'aspect renflé, relativement proche de la forme 3. Elle s'en distingue cependant par un épaississement de sa partie médiane, qui dans tous les cas ne dépasse pas le tiers de la largeur des extrémités (d'où la formule: larg. max.: $\leq 4 / 3$ des extrémités). Les exemplaires connus, attestés uniquement dans la version courte, se rapportent à une seule épave (SM6). Toutefois, leur aspect renflé est si caractéristique qu'il doit correspondre à une volonté délibérée du forgeron; ces pains méritent donc de constituer une forme à part entière. Cet aspect renflé paraît cependant lié à une forme courte, il y a peu de chance en conséquence de la découvrir un jour dans la version moyenne ou longue.

La forme 6 correspond à un pain aplati, sorte de plaque très large aux extrémités parfois arrondies. La largeur moyenne est de $10 \mathrm{~cm}( \pm 0,5)$ et la hauteur de $3,7 \mathrm{~cm}$ $( \pm 0,7 \mathrm{~cm})$. Comme la précédente, la forme 6 est attestée dans une seule épave de Camargue (SM6) et uniquement en module court. On en connaît d'autres exemplaires dans un gisement des Bouches de Bonifacio, Corse-du-Sud (Zwicker, 1996) et, surtout, dans l'épave Mateille A à Gruissan, Aude (Solier dir., 1981, p. 206, fig. 81), où se situait vraisemblablement un des ports d'embarquement de ces matières premières.

Les dimensions indiquées dans le tableau I correspondent aux valeurs moyennes, indépendamment des épaves d'où proviennent ces barres. À titre d'exemple, sur SM2, la longueur des grandes barres de forme 1L varie entre $96 \mathrm{~cm}$ et $115 \mathrm{~cm}$; le plus grand nombre (soit 70 sur 97 exemplaires mesurés) est compris entre $105 \mathrm{~cm}$ et $110 \mathrm{~cm}$. Sur SM8, les trois quarts des barres de forme 2 mesurent entre $48 \mathrm{~cm}$ et $55 \mathrm{~cm}$ (forme 2M). Les mêmes variations s'observent sur les pains trapus de forme 4. Ainsi, sur SM9, la moyenne des longueurs s'établit autour de 25-30 cm. Dans SM24 enfin, la longueur des grandes barres de forme $4 \mathrm{~L}$ varie du simple au double, voire au triple, bien que les sections restent à peu près constantes. De la même manière, les écarts de poids peuvent être importants d'un exemplaire à l'autre au sein d'un même groupe (tabl. I). L'état de conservation est certainement une des explications à ces variations, car certaines barres sont profondément rongées par endroits. Par ailleurs, le poids d'une barre dépend aussi de la matière première, donc du poids originel du massiot ou du tronçon de massiot travaillé par le forgeron ${ }^{8}$, et, pourquoi pas, du tour de main de ce dernier. Sur SM9 par exemple, les pains de forme 4C sont issus de deux ateliers distincts, différenciés par les timbres qu'ils portent, S//LEPIDI//N pour les uns et IVL//EROTIS pour les autres. Le premier fabrique des pièces plutôt massives et plus lourdes que le second, dont les exemplaires offrent un aspect plus fin. Il est donc illusoire de vouloir rechercher un poids moyen pour chaque type de barre et, par conséquent, un éventuel calibrage fondé sur la livre romaine, comme il en existait pour les lingots de plomb à la même époque ${ }^{9}$ (Domergue, Liou, 1997, p. 17-20). La variété des formes semble avoir été dictée par un certain souci de normalisation, davantage pour répondre à l'utilisation ultérieure des barres que pour observer un poids standard. On pense en particulier aux longues barres de formes 1L et 4L qui pourraient avoir été destinées à la fabrication de bandages de roues de char par exemple, voire d'armes, d'ancres de navire ou d'autres objets de grandes dimensions. Mais il est bien difficile d'attribuer une destination spécifique à chaque type de barre. Les pains de formes 3C et 4C, et leurs dérivés (F5 et F6), convenaient sans doute à la fabrication d'objets de petit ou moyen format. On peut penser aussi que le format des barres était dicté par des impératifs de stockage et/ou de commercialisation.

\section{LES ESTAMPILLES}

La présence d'estampilles sur les barres de fer des épaves de Camargue est très fréquente, mais ne semble pas avoir été systématique. Par exemple, aucune des barres de forme 2 récupérées sur SM8 et SM25 n'en présente. Sur les autres épaves, la majorité des barres est estampillée, mais la corrosion et/ou les concrétions empêchent d'établir des pourcentages fiables entre celles qui l'étaient effectivement et celles qui ne l'étaient pas, globalement et au sein d'un même atelier. Là où l'estampillage peut être observé, il se

8. Le fer obtenu par le procédé de réduction directe, seule méthode en usage dans l'Antiquité, présentait un aspect spongieux; c'est le massiot ou loupe de fer. Suivait un travail de forgeage qui devait respecter deux phases principales, l'épuration d'abord, le martelage ensuite et, dans le cas des plus grandes barres probablement, la soudure, afin de donner à la barre la forme et la longueur voulues.

9. On notera toutefois que le poids maximal d'une longue barre de SM24 est proche de 100 livres romaines (voir supra, p. 248). 


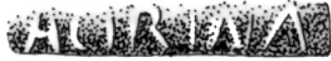

1

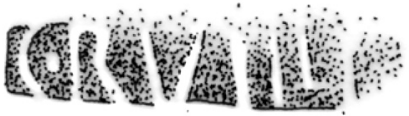

3

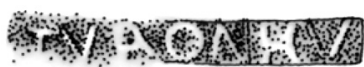

2

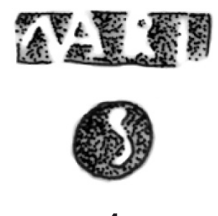

4
Fig. 5 - Exemples de timbres et associations de timbres des barres de fer antiques de Camargue: 1, Acirina (Arles, Trinquetaille); 2, Turonicu (Arles, Trinquetaille) ; 3, C. Rutili (SM6) ; 4, Mari//S (SM3) (relevés C. Rico, UTAH).

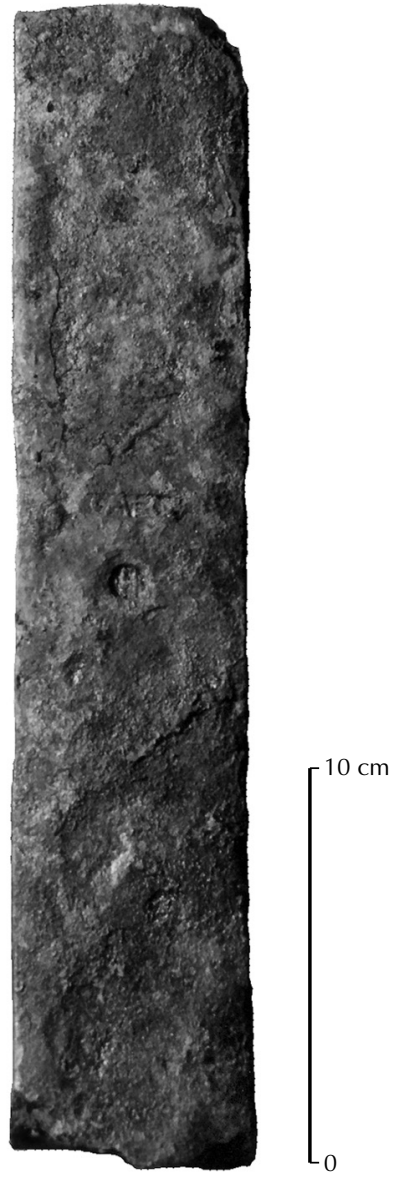

Fig. 6 - Barre de forme $4 C$ avec estampilles centrales CAECI//H (épave SM9) (cliché C. Rico, UTAH). présente sous la forme d'un ou de plusieurs timbres en creux, renfermant des initiales ou des noms plus ou moins abrégés en relief (fig. 5 et 6 ). Aucune incision pratiquée à froid (graffito) n'a été relevée. Cette absence est peut-être à mettre sur le compte de l'oxydation qui aurait fait disparaître des inscriptions par essence superficielles, comme on en connaît sur les lingots de plomb et de cuivre. Sur ces derniers, il s'agit bien souvent de marques pondérales qui indiquent le dépassement de poids par rapport à la norme. Or, si l'on accepte que les différents types de barres de fer ne suivaient pas des poids standards, leur absence n'est pas étonnante ici.

Ce sont donc essentiellement des cachets en creux qui apparaissent, réalisés par des poinçons-matrices. Ont-ils été apposés à froid ou à chaud? La question n'est pas secondaire, tant la réponse qu'on y apportera conditionnera leur interprétation. Imprimés à chaud, les timbres sont la signature des fabricants; à froid, ils peuvent tout aussi bien avoir été apposés dans un deuxième temps, hors de la forge, et ils se rapportent alors à la phase de commercialisation. Dans ce cas, ils ne signaleraient plus les ateliers mais donneraient les noms des commerçants du fer. Leur aspect ne permet pas de trancher définitivement entre une impression à froid et une impression à chaud; il dépend certainement aussi de la nature même du métal. Si certains poinçons sont profondément imprimés, ce n'est pas le cas de tous. Mais, en revanche, tous sont bien formés et peu, en définitive, révèlent une impression superficielle qui indiquerait que les matrices ont été appliquées sur un métal froid. Enfin, toutes les estampilles ont été disposées régulièrement et avec soin, toujours au même emplacement; une même estampille n'apparaît jamais à deux emplacements différents sur deux barres de même forme (tabl. II). La régularité de l'estampillage, la forme des timbres et le fait que ceux-ci sont toujours imprimés avec soin, tout conduit à voir dans ces estampilles les marques des fabricants, à l'égal de celles qui, à la même période, sont moulées sur les lingots de plomb. De cette façon, les ateliers pouvaient être identifiés par les noms de leurs propriétaires, pratique habituelle à l'époque romaine, et pas seulement pour le métal ${ }^{10}$.

10. Ce n'est pas le lieu ici de s'engager dans la discussion qu'entraîne systématiquement l'étude des marques de l'instrumentum domesticum autour de l'identité et de la fonction exacte des personnages qui apparaissent sur les timbres. Les problèmes soulevés par ces inscriptions sont multiples, quels que soient les supports et les matériaux sur lesquels elles apparaissent, et ont été très bien analysés par C. Bémont (1991, p. 135-136). On se limitera ici à souligner que le timbre, en tant que marque de fabrique, témoigne de l'existence d'un responsable des objets concernés (id., ibid., p. 136). Préciser à quel niveau de la production se situe cette responsa-bilité est chose peu aisée, en raison de la concision des estampilles. 
Tabl. II - Agencement des estampilles sur les barres de fer antiques des Saintes-Maries-de-la-Mer (les flèches symbolisent le long côté des barres et, par conséquent, le sens dans lequel doit se faire la lecture des timbres).

\begin{tabular}{|c|c|c|c|c|}
\hline Série & Nom & Forme & Position & Épave \\
\hline 1 & $\begin{array}{l}\text { C. RVTILI } \\
\text { CAVLI (?) } \\
\text { I[---]CI ; CAECI ; T. COR ; T. AFRAN }\end{array}$ & $\begin{array}{l}2 \mathrm{M} \\
4 \mathrm{C} \\
4 \mathrm{C}\end{array}$ & $\begin{array}{l}\text { extrémité droite } \\
\text { centrale }\end{array}$ & $\begin{array}{l}\text { SM6 } \\
\text { SM9 } \\
\text { SM9 }\end{array}$ \\
\hline $2 \vec{\square}$ & [---]//[.]AT[ & $2 \mathrm{M}$ & centrale & SM6 \\
\hline $3 \vec{\square}$ & [----]MI//[----]Ml & $2 \mathrm{M}$ & centrale & SM9 \\
\hline 4 & FAL (?)//CAECl & $4 \mathrm{C}$ & centrale & SM9 \\
\hline 5 & $\begin{array}{l}\mathrm{CAECI} / / \mathrm{H} \\
\mathrm{MARI} / / \mathrm{S}\end{array}$ & \begin{tabular}{|c|}
$4 \mathrm{C}$ \\
$1 \mathrm{ou} 2$
\end{tabular} & centrale & $\begin{array}{l}\text { SM9 } \\
\text { SM3 }\end{array}$ \\
\hline 6 & (timbres illisibles) & $2 \mathrm{M}$ & $\begin{array}{l}\text { centrale ou } \\
\text { extrémité }\end{array}$ & SM9 \\
\hline $7 a$ & MAXIMI//MAXIMI//[---] & $1 \mathrm{~L}$ & centrale & SM10 \\
\hline $7 \mathrm{~b}$ & Q CATO//Q CATO//TEREN & $1 \mathrm{~L}$ & centrale & SM10 \\
\hline 8 & \begin{tabular}{|l} 
FVLVIOR//FVLVIOR \\
MANI ?//[MANI ?] \\
(timbres illisibles)
\end{tabular} & $\begin{array}{l}4 \mathrm{C} \\
4 \mathrm{~L} \\
3 \mathrm{~L}\end{array}$ & $\begin{array}{l}\text { centrale } \\
2 \text { extrémités } \\
\text { centrale }\end{array}$ & $\begin{array}{l}\text { SM10 } \\
\text { SM24 } \\
\text { SM24 }\end{array}$ \\
\hline 9 & $\begin{array}{l}\text { IVL//EROTIS (redoublée) } \\
\text { (timbres illisibles) }\end{array}$ & $\begin{array}{c}1 \mathrm{~L}-4 \mathrm{C} \\
4 \mathrm{C} \\
4 \mathrm{~L}\end{array}$ & $\begin{array}{c}\text { extrémité supérieure } \\
\text { extrémité }\end{array}$ & $\begin{array}{l}\text { SM2 } \\
\text { SM9 } \\
\text { SM24 }\end{array}$ \\
\hline 10 & $\begin{array}{l}\text { S//LEPIDI//N } \\
\text { (ancora) }\end{array}$ & $4 \mathrm{C}$ & 2 extrémités & SM2 \\
\hline
\end{tabular}

Ces noms, quand ils sont déchiffrables, tâche peu aisée tant la corrosion a souvent irrémédiablement dégradé des lettres qui ne dépassent pas 5 à $6 \mathrm{~mm}$ de hauteur, sont indiqués au génitif et sont peu abrégés. On ne trouve pas d'abréviation complète de duo ou tria nomina; seuls les prénoms, comme c'est l'usage, sont réduits à leur initiale. La partie terminale des gentilices est généralement abrégée: FVLVIOR pour Fuluiorum (SM10), TEREN pour Terentii (SM10), IVL pour Iulii (SM2 et SM9), AFRAN pour Afranii (SM9), COR pour Cornelii (SM9), et les cognomina sont toujours écrits en toutes lettres.

Nous avons, à ce jour, répertorié dix systèmes d'estampillage différents, du plus simple au plus complexe; ils vont du timbre unique, le plus souvent imprimé au centre de la barre, à des agencements divers, associant plusieurs poinçons, de deux à quatre, de forme rectangulaire ou circulaire. Ces dix séries sont présentées dans le tableau II.

Plusieurs séries, 3, 7a et 7b, 8 et 9, présentent les mêmes timbres imprimés deux fois, selon la même disposition: ainsi, FVLVIOR dans SM1O; dans cette même épave, Q. CATO sur une série de barres de forme 1 et MAXIMI sur une autre. Dans l'un et l'autre cas, ils sont complétés par un timbre, rectangulaire lui aussi, disposé perpendiculairement: TEREN dans le premier, mais illisible dans le second. Dans SM2 et SM9, des barres de formes 2 et 4 présentent le même système d'estampillage qui redouble le couple IVL dans un poinçon circulaire et EROTIS dans un timbre rectangulaire, identifiant un producteur du nom de Iulius Eros. Quelle que soit leur forme, ces timbres, associés sur une même barre, constituent, une fois juxtaposés, l'estampille du fabricant. Les séries 5 et 10 pourtant semblent quelque peu différentes. Dans l'une et l'autre, elles associent un timbre rectangulaire portant un cognomen au génitif et un poinçon circulaire (série 5), voire deux (série 10), circonscrivant une simple initiale. Celle-ci pose problème. Si les noms en toutes lettres sont ceux des fabricants, que signifient alors le $\mathrm{H}$ associé au cognomen CAECI sur SM9 et le $\mathrm{S}$ associé à MARI (fig. 5, no 4) sur certaines barres de forme 1 (ou 2) de $S M 3^{11}$ ? De même, comment développer les deux lettres $\mathrm{S}$ et $\mathrm{N}$ qui encadrent, sur les barres de forme 4 de SM2, le surnom LEPIDI ${ }^{12}$ ? Nous n'avons pas encore de réponse. Les poinçons circulaires ont-ils été imprimés dans un deuxième temps ? La régularité du marquage, la disposition invariable des différentes marques sur toutes les barres, enfin la cohérence des agencements ne permettent pas de le penser. La même question se pose pour les séries $7 \mathrm{~b}$ et 8 . Dans la première, un timbre circulaire, dont l'inscription a disparu, est associé à l'ensemble Q. CATO//TEREN. Dans la seconde, la même estampille FVLVIOR encadre un poinçon circulaire très corrodé, impossible à déchiffrer.

11. Tout récemment, P. A. Gianfrotta ( I relitti de la Camargue e Sextus Marius Hispaniarum ditissimus [Tacite, Annales, VI, 19, 1]", RSLig, LXX, 2004 [2005], p. 161-176) a proposé de développer Mari//S en $S$ (extus) Mari(us), qui désignerait, dans l'hypothèse présentée longuement par l'auteur, le richissime Sextus Marius de Tacite (Annales, VI, 19, 1), propriétaire au temps de Tibère de mines dans la province de Bétique. C'est peu probable. On fera remarquer d'abord que la lecture des timbres est MARI//S et non pas S//MARI, ce qui n'est pas la même chose. D'autre part, on ne peut pas interpréter, nous semblet-il, MARI//S sans tenir compte des estampilles S// LEPIDI//N et CAECI//H, présentes sur d'autres barres. Caecus et Lepidus sont des cognomina, Marus doit aussi en être un, même si le gentilice Marius est toujours possible. De plus, ces cognomina sont eux aussi suivis d'un poinçon circulaire renfermant une seule lettre $(\mathrm{N}, \mathrm{H})$, dont la signification, comme celle du $\mathrm{S}$ dans MARI//S, reste mystérieuse. L'hypothèse de P. A. Gianfrotta touche par ailleurs à la question de l'origine géographique de certaines des cargaisons de barres de fer des Saintes-Maries-de-la-Mer: soit il faut la chercher dans le sud de l'Espagne, soit Sextus Marius possédait aussi des entreprises métallurgiques en Gaule méridionale (p. 173). Mais, en l'absence de données véritablement nouvelles (en particulier archéométriques), l'interprétation et les hypothèses de P. A. Gianfrotta nous semblent prématurées. 12. Le problème est d'autant plus aigu qu'une barre de la même épave présente le timbre rétrograde LEPIDI, encadré par deux poinçons ovales présentant l'un et l'autre une haste en relief. 
Les raisons du choix de tel ou tel système d'estampillage, simple ou complexe, nous échappent. Chaque atelier avait-il son propre système de marquage? Une barre de forme 1L, remontée en août 2005 de l'épave SM10, pourrait apporter une réponse en ce sens. Elle présente, malgré l'état dégradé des inscriptions, l'association de trois timbres, disposés en U (série 7a), Q. CATO//TEREN//MAXIMI. Connus individuellement sur des barres de la même épave, Q. Terentius Cato et Maximus utilisent un système d'estampillage identique. Celui-ci apparaît alors comme la marque propre de l'atelier, qui est en l'occurrence l'association de deux individus. En allait-il de même pour tous les autres systèmes d'estampillage? Ce n'est pas si simple. Le timbre unique (série 1) est utilisé par plusieurs producteurs que rien ne permet de relier entre eux. De la même manière, les séries 5 et 8 comportent chacune deux noms différents, Caeci et Mari dans la première, Fuluior(um) et Mani(i) dans la seconde. Mais rien ne dit que Caecus et Marus (ou Marius?) d'un côté, les Fuluii et Manius de l'autre, étaient associés, à l'instar de Q. Terentius Cato et de Maximus, d'autant plus que les barres marquées à leurs noms proviennent d'épaves différentes (tabl. II).

Les estampilles sur les barres de fer des épaves romaines des Saintes-Maries-de-la-Mer gardent donc une part de mystère. L'étude onomastique fournit certes quelques pistes et nous oriente vers le sud de la Gaule, où les gentilices identifiés sur ces barres, Rutilius, Terentius, Fulvius, sont bien représentés, en particulier à Narbonne. C'est à Narbonne aussi que l'on rencontre d'autres noms, moins communs, tels Afranius et l'hypothétique Falius (Long et al., 2002, p. 187). Or Narbonne, dont on connaît la place dans le commerce maritime à la charnière de la République et de l'Empire, pourrait aussi être le débouché d'une production qui prend tout son essor à cette période: le fer de la Montagne Noire. Mais tout cela a besoin d'être confirmé. L'étude qui suit est un premier pas dans ce sens.

\section{ÉTUDE PALÉOMÉTALLURGIQUE DES BARRES DE FER}

Les cargaisons de barres de fer des épaves antiques des Saintes-Maries-de-la-Mer ayant été évaluées, pour certaines d'entre elles, à plusieurs dizaines de tonnes (Long, 1997), il s'est avéré nécessaire, dans le cadre d'une étude préliminaire, d'effectuer une sélection rigoureuse des exemplaires, en vue de leur analyse: le nombre d'échantillons devait être minimal ${ }^{13}$, tout en permettant de répondre à un maximum de questions ${ }^{14}$.

Nous avons donc choisi d'étudier six barres en détail: SM2-96-K-46, SM10-98-8, SM9-99-248, SM9-98-50-82, SM9-55 et SM2-61. Elles proviennent de trois épaves différentes (SM2, SM9 et SM10), sur les sept qui en renfermaient et qui avaient fait l'objet d'expertises au moment de l'étude. Les épaves SM2, SM9 et $S M 10$ se situent respectivement au premier quart du I ${ }^{\mathrm{er}}$ s. apr. J.-C., entre l'époque augustéenne et celle de Claude, et enfin au I ${ }^{\text {er }}$ s. apr. J.-C. Ces barres sont de trois formes différentes (1L, 2M et 4C), deux d'entre elles sont estampillées (fig. 11).

\section{LE MÉTAL DES BARRES}

L'observation du métal ${ }^{15}$ a révélé l'hétérogénéité de sa composition et de sa microstructure au sein d'une même barre, et cela pour tous les échantillons étudiés. Par exemple, dans une partie de la barre SM9-98-50-82 (forme 4C), nous observons un acier dont la microstructure comporte des plaquettes claires de ferrite (aiguilles dans le plan de coupe), soit délimitant les joints fantômes de l'austénite, soit noyées dans des colonies sombres de perlite (structure biphasée de lamelles alternées de ferrite et de cémentite $\mathrm{Fe}_{3} \mathrm{C}$ ) ; dans d'autres zones, la microstructure est différente: de type perlitique globulaire, elle témoigne d'un maintien plus long à une température élevée (fig. 7a et b).

Il s'agit, dans les deux cas, d'acier hypoeutectoöde (teneur en carbone inférieure à $0,8 \%$ ) ; les proportions et distributions de la ferrite et de la cémentite, parfois très différentes,

13. Cette étude, entreprise sur la suggestion de Francis Tollon, a été réalisée dans le cadre du DESS « Méthodes scientifiques et techniques en archéologie » de l'université de Bourgogne (Coustures, 2001) ; la durée nécessairement limitée de l'étude explique que nous n'ayons pu analyser qu'un faible nombre d'échantillons. Des travaux comparables sont actuellement en cours sur d'autres barres des Saintes-Maries-dela-Mer et la recherche d'éléments traceurs se poursuit pour d'autres centres sidérurgiques antiques.

14. Les prélèvements ont été effectués sur du métal sain et, par souci de représentativité, plusieurs sections polies ont été réalisées et étudiées pour chaque barre. Les précautions d'usage inhérentes aux procédés techniques et aux méthodes d'analyse sont détaillées dans Coustures, 2001 et ont été respectées. On se reportera aussi à Ploquin, 2004a et 2004b.

15. Les études métallographiques ont été réalisées au Laboratoire «Interfaces et Matériaux» de l'ENSIACET (INP Toulouse). Les sections polies des prélèvements sur barres ont été soumises à une attaque chimique au réactif Nital $5 \%$ (solution diluée de quelques pourcentages d'acide nitrique dans l'alcool) pour révéler la microstructure du métal, et ont été examinées au microscope métallographique. 


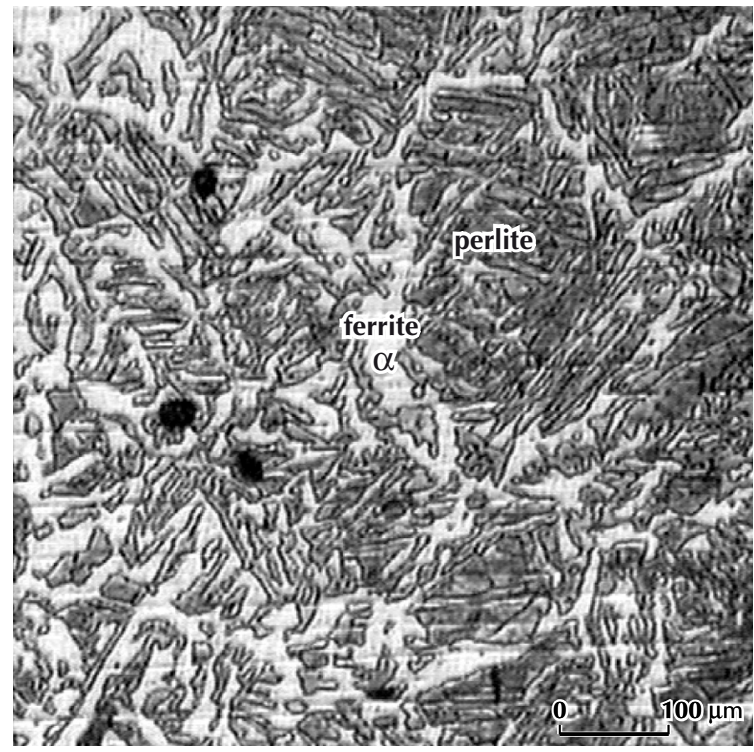

a

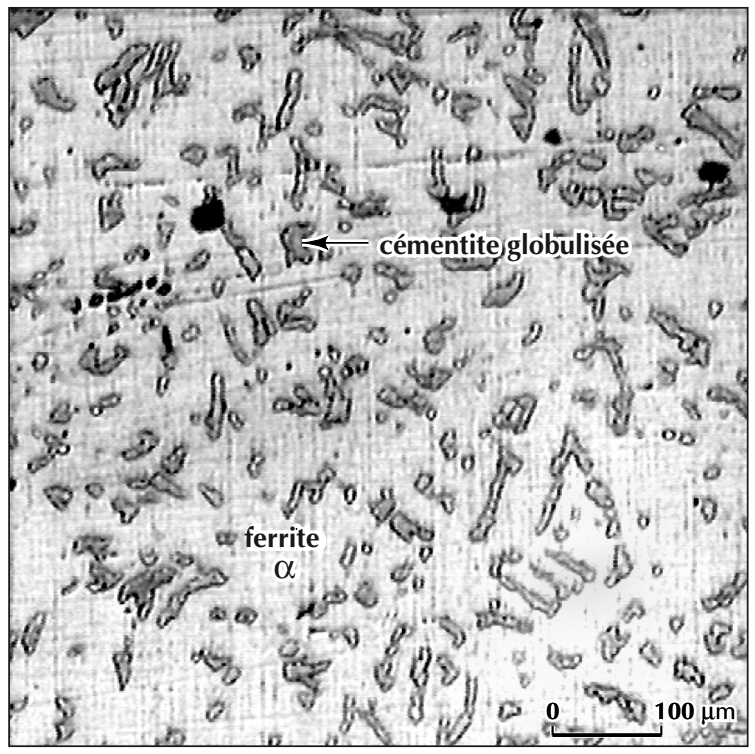

b

Fig. 7 - Photographies au microscope métallographique de la microstructure des deux types d'acier d'une barre de fer : a, association de ferrite $\alpha$ et de perlite (lamelles alternées de cémentite, en gris, et de ferrite, en blanc) ; b, microstructure perlitique globulaire (clichés M.-P. Coustures, UTAH).

traduisent l'hétérogénéité de la composition du métal, qui peut résulter soit de l'utilisation d'un massiot hétérogène, soit de celle de plusieurs massiots. Mais la présence d'aciers d'histoires thermiques différentes suggère, comme cela a pu être montré pour d'autres lingots, que les barres ne sont pas nécessairement issues du forgeage d'un seul et même massiot, mais plutôt de l'assemblage par forgeage-soudage de massiots différents. Cette hypothèse est confirmée par l'identification d'une soudure en $\mathrm{S}$ sur une barre de fer de forme 1 dans l'épave SM2 (Long et al., 2002, p. 164).

\section{LES INGLUSIONS DE SGORIE}

L'épuration par martelage de la loupe de fer n'étant jamais parfaite, des scories sont présentes sous forme d'inclusions dans l'acier des barres.

\section{COMPOSITIONS MINÉRALOGIQUES}

Les caractéristiques minéralogiques des inclusions de scorie sont les mêmes, quelle que soit la barre étudiée. On retrouve, en effet, dans les six exemplaires observés les deux types d'inclusions de scorie déjà mis en évidence dans d'autres barres de fer (Hedges, Salter, 1979) et dans des objets finis (Dillmann, 1998; Dillmann, Balasubramaniam, 2001 ; Renoux et al., 2001 ; Serdon, Fluzin, 2002). Ce sont des inclusions de scorie entièrement vitreuses et des inclusions à cristaux de wüstite dans une matrice vitreuse (fig. $8 \mathrm{a}$ et b).

En revanche, la quantité et la dimension de ces inclusions varient d'une barre à l'autre. La barre SM2-96-K-46 se distingue ainsi par une absence presque totale d'inclusions de scorie, mais ces dernières, quand elles sont présentes, sont de très petite taille $(<150 \mu \mathrm{m}$ de largeur), y compris dans le plan de plus grand aplatissement de la barre dans lequel elles offrent la surface analysable la plus importante. À l'opposé, la barre SM9-99-248 est remarquable par la quantité et les dimensions importantes des scories que l'on y rencontre.

\section{COMPOSITIONS CHIMIQUES}

Les résultats des analyses chimiques des inclusions de scorie sont présentés dans les tableaux III et IV ${ }^{16}$.

Les inclusions de scorie à cristaux de wüstite et matrice vitreuse sont généralement considérées comme résultant d'une épuration imparfaite, alors que les inclusions entièrement vitreuses se formeraient à partir d'ajouts de forge

16. Les analyses ont été effectuées au Laboratoire de géochimie de Toulouse. On a utilisé un laser combiné avec une torche à plasma à couplage inductif et un spectromètre de masse (LA-ICP-MS). En raison du diamètre du faisceau laser utilisé, entre 150 et $300 \mu \mathrm{m}$, les inclusions de scorie de taille inférieure n'ont pu être analysées; c'est le cas de la barre SM2-99-K-46. L'ablation a duré 160 secondes. 
Tabl. III - Compositions en éléments majeurs des inclusions de scorie entièrement vitreuses de barres de fer des Saintes-Maries-dela-Mer (unité : pourcentage poids oxydes) : Al, aluminium ; Ba, baryum ; Ca, calcium ; Cr, chrome ; Cu, cuivre ; Fe, fer ; $K$, potassium ; Mg, magnésium ; Mn, manganèse ; $\mathrm{Na}$, sodium ; Ni, nickel ; O, oxygène ; $P$, phosphore ; Si, silicium ; Ti, titane.

\begin{tabular}{|l|c|c|c|c|c|c|c|c|c|c|c|c|c|c|c|}
\hline \multicolumn{1}{|c|}{ Échantillon } & $\mathbf{N a}_{\mathbf{2}} \mathbf{O}$ & $\mathbf{M g O}$ & $\mathbf{A l}_{\mathbf{2}} \mathbf{O}_{\mathbf{3}}$ & $\mathbf{S i O}_{\mathbf{2}}$ & $\mathbf{P}_{\mathbf{2}} \mathbf{O}_{\mathbf{5}}$ & $\mathbf{K}_{\mathbf{2}} \mathbf{O}$ & $\mathbf{C a O}$ & $\mathbf{T i O}_{\mathbf{2}}$ & $\mathbf{C r}_{\mathbf{2}} \mathbf{O}_{\mathbf{3}}$ & $\mathbf{M n O}$ & $\mathbf{F e O}$ & $\mathbf{N i O}$ & $\mathbf{C u O}$ & $\mathbf{B a O}$ & $\mathbf{T o t a l}$ \\
\hline SM2-61-2 & 0,56 & 0,64 & 5,80 & 34,04 & 0,67 & 3,10 & 7,36 & 0,02 & 0,00 & 1,57 & 46,21 & 0,00 & 0,01 & 0,07 & 100,04 \\
\hline SM2-61-1 & 0,49 & 1,23 & 5,65 & 36,55 & 0,37 & 2,71 & 7,25 & 0,17 & 0,03 & 3,10 & 41,92 & 0,03 & 0,00 & 0,13 & 99,66 \\
\hline SM9-98-50-82-1 & 0,66 & 0,60 & 5,25 & 35,33 & 0,56 & 2,00 & 8,91 & 0,01 & 0,00 & 4,51 & 42,30 & 0,00 & 0,07 & 0,00 & 100,21 \\
\hline SM9-98-50-82-3 & 0,56 & 1,56 & 5,88 & 34,39 & 0,71 & 1,86 & 8,42 & 0,15 & 0,05 & 11,16 & 35,42 & 0,00 & 0,01 & 0,01 & 100,19 \\
\hline SM9-98-50-82-4 & 0,74 & 1,51 & 6,01 & 35,86 & 0,25 & 2,33 & 8,33 & 0,15 & 0,01 & 9,67 & 35,05 & 0,00 & 0,08 & 0,00 & 100,00 \\
\hline SM9-98-50-82-5 & 1,07 & 2,08 & 9,55 & 53,20 & 0,00 & 3,92 & 12,35 & 0,29 & 0,00 & 13,13 & 4,22 & 0,05 & 0,00 & 0,07 & 99,94 \\
\hline SM10-98-8-2 & 0,41 & 1,42 & 5,43 & 31,68 & 0,60 & 1,75 & 2,60 & 0,06 & 0,00 & 1,50 & 53,96 & 0,00 & 0,00 & 0,00 & 99,43 \\
\hline SM10-98-8-4 & 0,92 & 3,14 & 11,89 & 58,44 & 0,03 & 4,34 & 5,21 & 0,41 & 0,07 & 0,48 & 14,04 & 0,01 & 0,09 & 0,00 & 99,08 \\
\hline SM9-99-248-3 & 0,15 & 0,28 & 3,47 & 30,27 & 0,61 & 0,98 & 2,86 & 0,14 & 0,00 & 1,43 & 59,91 & 0,05 & 0,00 & 0,03 & 100,18 \\
\hline SM9-99-248-2 & 0,16 & 0,29 & 2,90 & 29,53 & 0,79 & 1,10 & 2,97 & 0,18 & 0,03 & 1,46 & 60,71 & 0,01 & 0,04 & 0,00 & 100,18 \\
\hline SM9-99-248-1 & 0,25 & 0,14 & 4,54 & 24,95 & 1,05 & 1,95 & 3,95 & 0,41 & 0,00 & 0,89 & 61,92 & 0,00 & 0,00 & 0,00 & 100,05 \\
\hline SM9-55-4 & 0,01 & 1,69 & 1,91 & 29,51 & 0,05 & 0,28 & 2,93 & 0,10 & 0,05 & 3,52 & 60,35 & 0,00 & 0,12 & 0,00 & 100,53 \\
\hline SM9-55-3 & 0,45 & 0,00 & 16,21 & 35,05 & 1,90 & 5,86 & 14,20 & 0,27 & 0,00 & 0,75 & 24,27 & 0,00 & 0,00 & 0,00 & 98,99 \\
\hline
\end{tabular}

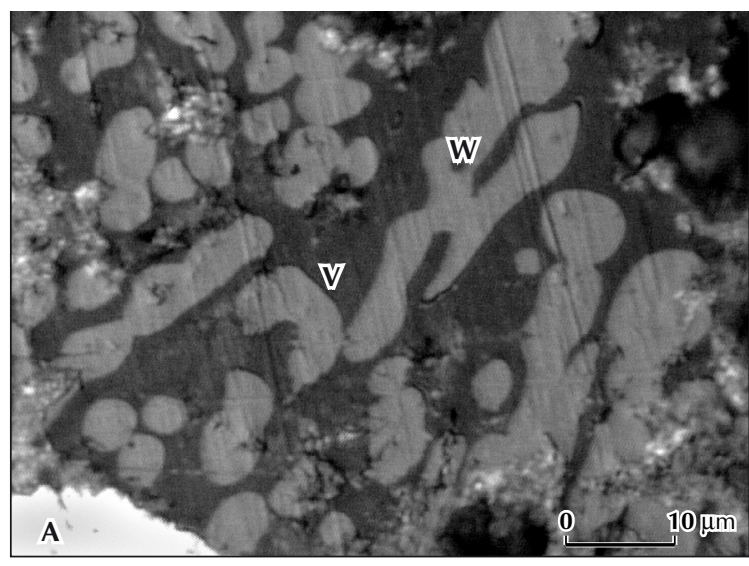

a

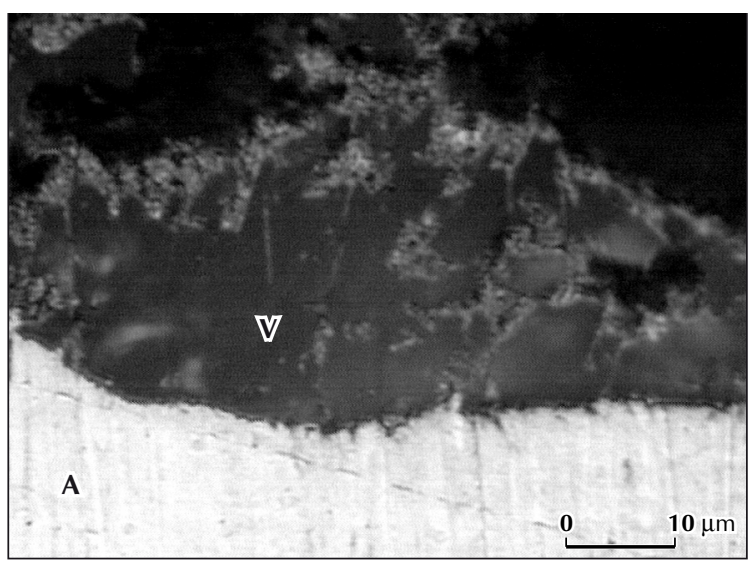

b

Fig. 8 - Photographies au microscope métallographique de la texture des deux types d'inclusions d'une barre de fer : a, inclusions à cristaux de wüstite dans une matrice vitreuse ; $b$, inclusions de scorie entièrement vitreuses ; A, acier ; V, verre; W, wüstite (clichés M.-P. Coustures, UTAH).

(Renoux et al., 2001; Serdon, Fluzin, 2002; Mangin dir., 2004). Ces dernières, résultant essentiellement de la fusion d'un mélange de chaux, sable et argile, sont pauvres en fer (Leblanc, 2002) ; par exemple, Renoux et al. (2001) indiquent une teneur maximale de $10 \%$ en poids de $\mathrm{FeO}$ pour ce type d'inclusions.

La plupart des inclusions de scorie entièrement vitreuses des barres des épaves de Saintes-Maries-de-la-Mer renferment plus de $35 \%$ de FeO (tabl. III). Il est donc exclu que de telles inclusions aient pu se former à partir d'ajouts de forge; elles correspondraient, comme les inclusions à cristaux de wüstite, à des résidus de scorie de réduction. Toutefois, la teneur en fer dans ces inclusions ne serait pas suffisamment élevée pour induire la formation des dendrites de wüstite (Mahé-Le Carlier, 1997).

De plus, les analyses chimiques montrent qu'au sein d'une même barre de fer, il existe une homogénéité de composition en éléments en trace quasi parfaite entre les inclusions entirement vitreuses renfermant plus de $35 \%$ de $\mathrm{FeO}$ et les inclusions à matrice vitreuse et cristaux de wüstite 
Tabl. IV - Compositions en éléments en trace des inclusions de scorie de barres de fer des Saintes-Maries-de-la-Mer (unité : ppm) :

Ba, baryum ; Ce, cérium ; Cr, chrome ; Cs, césium ; Eu, europium ; Hf, hafnium ; La, lanthane ; Lu, lutécium ; Nd, néodyme ; Pr, praséodyme ; Rb, rubidium ; Sm, samarium ; Sr, strontium ; Th, thorium ; U, uranium ; V, vanadium ; Yb, ytterbium ; Zr, zirconium.

\begin{tabular}{|c|c|c|c|c|c|c|c|c|c|c|c|c|c|c|c|c|c|c|}
\hline Échantillon & La & $\mathrm{Ce}$ & $\operatorname{Pr}$ & Nd & Sm & Eu & $\mathrm{Yb}$ & Lu & $\mathrm{Cr}$ & $\mathbf{R b}$ & Cs & Ba & $\mathrm{Sr}$ & $\mathrm{Hf}$ & $\mathrm{Zr}$ & Th & u & v \\
\hline SM2-61-2 & 23,2 & 41,5 & 5,3 & 20,9 & 5,6 & 2,3 & 1,9 & n.d. & 16 & 118 & 3,08 & 811 & 251 & 2,71 & 81 & 7,89 & 3,18 & 24 \\
\hline SM2-61-1 & 23,3 & 42,5 & 5,6 & 21,8 & 5,5 & 2,6 & 2,1 & n.d. & 15 & 80 & 1,53 & 718 & 222 & 2,87 & 87 & 8,29 & 3,26 & 24 \\
\hline SM9-98-50-82-1 & 21,8 & 40,4 & 4,7 & 17,5 & 4,3 & 3,3 & n.d. & n.d. & 16 & 36 & n.d. & 233 & 140 & n.d. & 34 & 3,05 & 3,31 & 12 \\
\hline SM9-98-50-82-2 & 44,4 & 86,6 & 9,7 & 34,8 & n.d. & 6,9 & n.d. & n.d. & n.d. & 102 & n.d. & 551 & 311 & n.d. & 87 & 6,90 & 7,11 & 47 \\
\hline SM9-98-50-82-3 & 40,7 & 76,0 & 8,9 & 33,2 & 8,2 & 6,3 & n.d. & n.d. & n.d. & 43 & n.d. & 448 & 272 & n.d. & 71 & 5,56 & 5,87 & 41 \\
\hline SM9-98-50-82-4 & 35,7 & 66,5 & 7,8 & 28,8 & 6,8 & 5,4 & 2,4 & n.d. & 27 & 72 & 1,63 & 430 & 244 & 1,74 & 65 & 5,22 & 5,11 & 33 \\
\hline SM9-98-50-82-5 & 51,1 & 105,1 & 11,4 & 40,7 & n.d. & n.d. & n.d. & n.d. & n.d. & 127 & n.d. & 671 & 376 & n.d. & 91 & 6,89 & 7,56 & n.d. \\
\hline SM10-98-8-2 & 19,2 & 34,7 & 4,7 & 17,5 & n.d. & n.d. & n.d. & n.d. & 40 & 68 & 2,54 & 312 & 95 & n.d. & 82 & 5,33 & 3,02 & 41 \\
\hline SM10-98-8-4 & 22,6 & 41,0 & 5,3 & 20,5 & n.d. & n.d. & n.d. & n.d. & 72 & 84 & n.d. & 378 & 113 & n.d. & 97 & 5,97 & 3,41 & 64 \\
\hline SM9-99-248-5 & 194,2 & 335,8 & 48,5 & 211,8 & 50,3 & 11,8 & 21,7 & 3,3 & 120 & 45 & 4,59 & 1167 & 48 & 4,19 & 149 & 9,37 & 16,35 & 94 \\
\hline SM9-99-248-3 & 100,2 & 180,2 & 25,2 & 110,5 & 26,2 & 6,1 & 12,1 & 1,8 & 59 & 40 & 3,53 & 686 & 41 & 4,29 & 154 & 7,13 & 8,65 & 49 \\
\hline SM9-99-248-2 & 133,2 & 244,3 & 33,8 & 145,8 & 34,4 & 8,1 & 16,3 & 2,4 & 86 & 58 & 5,10 & 920 & 53 & 5,36 & 203 & 9,11 & 11,47 & 74 \\
\hline SM9-99-248-1 & 123,8 & 221,8 & 30,4 & 134,7 & 32,0 & 7,4 & 14,6 & 2,2 & 70 & 50 & 4,20 & 874 & 50 & 5,59 & 202 & 8,42 & 10,33 & 66 \\
\hline SM9-99-248-4 & 173,9 & 291,6 & 42,7 & 187,2 & 44,2 & 10,5 & 18,5 & 2,8 & 106 & 43 & 4,45 & 1000 & 40 & 3,00 & 115 & 7,26 & 13,93 & 90 \\
\hline SM9-55-4 & 52,1 & 104,7 & 12,6 & 49,3 & 10,9 & 5,1 & n.d. & n.d. & 100 & 127 & 5,74 & 590 & 303 & 7,70 & 270 & 15,55 & 4,56 & 89 \\
\hline SM9-55-3 & 52,6 & 108,3 & 12,5 & 49,6 & n.d. & n.d. & n.d. & n.d. & n.d. & 109 & n.d. & 584 & 308 & 7,30 & 265 & 15,61 & 4,71 & 61 \\
\hline SM9-55-1 & 14,2 & 29,9 & 3,4 & 13,2 & n.d. & n.d. & n.d. & n.d. & n.d. & 33 & n.d. & 160 & 87 & n.d. & 72 & 4,22 & 1,29 & 24 \\
\hline
\end{tabular}

échantillon/C1 chondrite

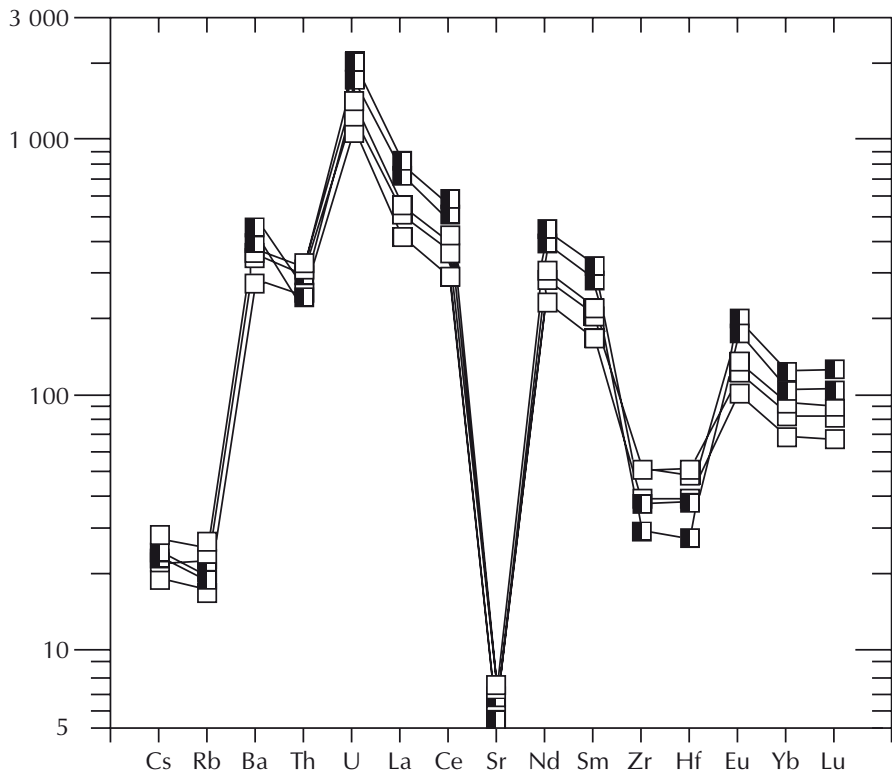

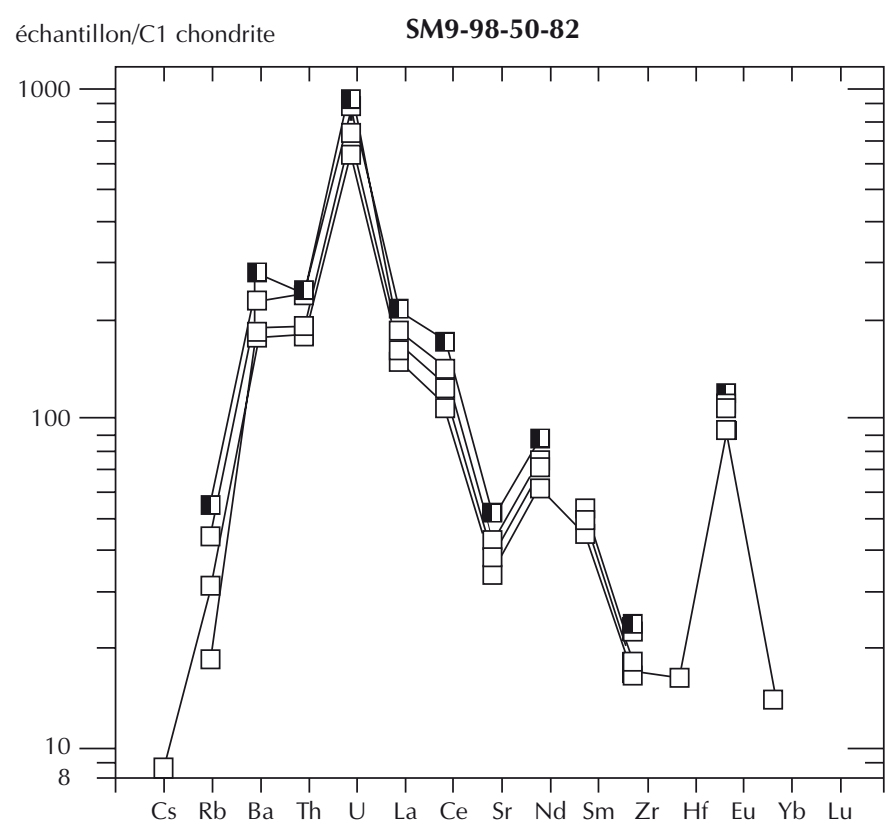

b

inclusions de scories entièrement vitreuses

inclusions de scories à matrice vitreuse et cristaux de wüstite

Fig. 9 - Distribution des concentrations en éléments en trace des inclusions de scorie : a, barre SM-9-99-248 ; b, barre SM9-98-50-82 (DAO M.-P. Coustures, UTAH). 
(fig. 9a et b). Des scories d'origines différentes (résidus de scorie de réduction ou résultats d'ajouts à la forge) présenteraient très vraisemblablement une différence de composition en éléments en trace.

Ainsi, pour procéder au test de provenance des barres de fer, seules les inclusions à cristaux de wüstite et celles qui sont entièrement vitreuses et renferment plus de $35 \%$ de $\mathrm{FeO}$ seront utilisées. En revanche, les inclusions pauvres en fer $(<20 \%$ en poids de $\mathrm{FeO})$, qui peuvent provenir pour une part d'ajouts de forge, sont éliminées.

\section{LES MARTYS, ORIGINE POSSIBLE DES BARRES. TEST DE L'HYPOTHÈSE}

Pour savoir si la Montagne Noire, et plus particulièrement le centre sidérurgique des Martys, est effectivement un lieu de production possible des barres des Saintes-Mariesde-la-Mer, nous avons utilisé la méthode de détermination de provenance des fers anciens fondée sur l'utilisation des compositions en éléments en trace des scories en inclusion dans ces barres.

Coustures et al. (2003) ont montré que certaines caractéristiques chimiques du minerai utilisé pour la réduction se transmettent à tous les produits scoriacés de la chaîne opératoire de fabrication d'un objet en fer et ce jusque dans les inclusions de scorie des barres mises en forme. Cela se traduit, pour un centre donné, par une forte corrélation entre les rapports de concentration de certains couples d'éléments en trace mesurés, d'une part, dans le minerai et, d'autre part, dans les scories de réduction et d'épuration et dans les inclusions de scorie des loupes de fer et barres de fer. Cette corrélation constitue une signature chimique du centre sidérurgique considéré et cette signature diffère d'un centre à l'autre ${ }^{17}$.

La détermination de la provenance des barres de fer des épaves de Saintes-Maries-de-la-Mer va donc reposer sur la comparaison avec l'atelier des Martys (Aude), la signature chimique de ce dernier ayant été établie par ailleurs (Coustures et al., 2003).

La signature chimique d'un centre sidérurgique est déterminée, pour certains couples d'éléments en trace, par un rapport constant de composition du minerai aux inclusions de scorie des barres de fer. Cette signature est matérialisée graphiquement, pour un couple d'éléments (Sr-Ba, par exemple), par une droite passant par l'origine,

17. L'autre centre sidérurgique ayant fait l'objet d'analyses est celui des Ferrys à La Bussière, Loiret (Coustures et al., 2003). la pente de cette droite étant caractéristique d'un lieu de production donné (Coustures et al., 2003). Si, pour tous les couples d'éléments en trace considérés, les points représentatifs de la composition des inclusions de scorie d'une barre se placent sur la droite représentant la signature chimique du centre sidérurgique des Martys, l'hypothèse de provenance sera validée. En revanche, si pour un ou plusieurs couples d'éléments en trace, ces mêmes points s'en écartent, l'hypothèse de provenance devra être rejetée. L'ensemble des résultats est présenté dans la figure 10.

Les points représentant les compositions en éléments en trace des inclusions de scorie des barres de fer SM2-61 et SM10-98-8 se placent, sur tous les graphiques, sur la droite de corrélation des Martys. Pour ces deux barres de fer, l'hypothèse de provenance peut être validée.

Hormis le cas du couple baryum-strontium (Ba-Sr), les points représentant la barre de fer SM9-98-50-82 se situent, sur tous les autres graphiques, à proximité de la droite de corrélation du bas fourneau des Martys. La composition en $\mathrm{Ba}$ et $\mathrm{Sr}$ des scories peut être influencée par celle de l'argile constituant le revêtement interne du bas fourneau (Crew, 2000). On observe donc, pour ce couple d'éléments en trace, une dispersion plus importante des points représentant les échantillons des Martys autour de la droite de corrélation. Cette dispersion n'est pas plus importante pour la barre de fer SM9-98-50-82 que pour les échantillons des Martys.

Les points représentant la composition en éléments en trace des inclusions de scorie des barres de fer SM9-99248 et SM9-55 s'écartent nettement de la droite de corrélation des Martys, excepté pour le couple lanthane-cérium (La-Ce). Toutefois, sur ce dernier graphique, les points représentant la barre de fer SM9-99-248 se distinguent fortement des autres par des concentrations absolues en La et Ce beaucoup plus importantes que dans les échantillons des Martys. Enfin, les points représentatifs des inclusions de scorie de ces deux barres de fer ne se situent pas sur une même droite dans les graphiques baryum-strontium et rubidium-césium (Ba-Sr et $\mathrm{Rb}-\mathrm{Cs}$ ).

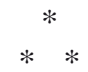

L'étude dont les résultats sont présentés ici doit être considérée comme préliminaire, puisqu'elle ne s'intéresse qu'à une des hypothèses de provenance envisagées et qu'elle ne concerne qu'un nombre limité de barres de fer. Elle ne prétend donc, en aucun cas, répondre de façon certaine et exhaustive à la question de l'origine des cargaisons des 

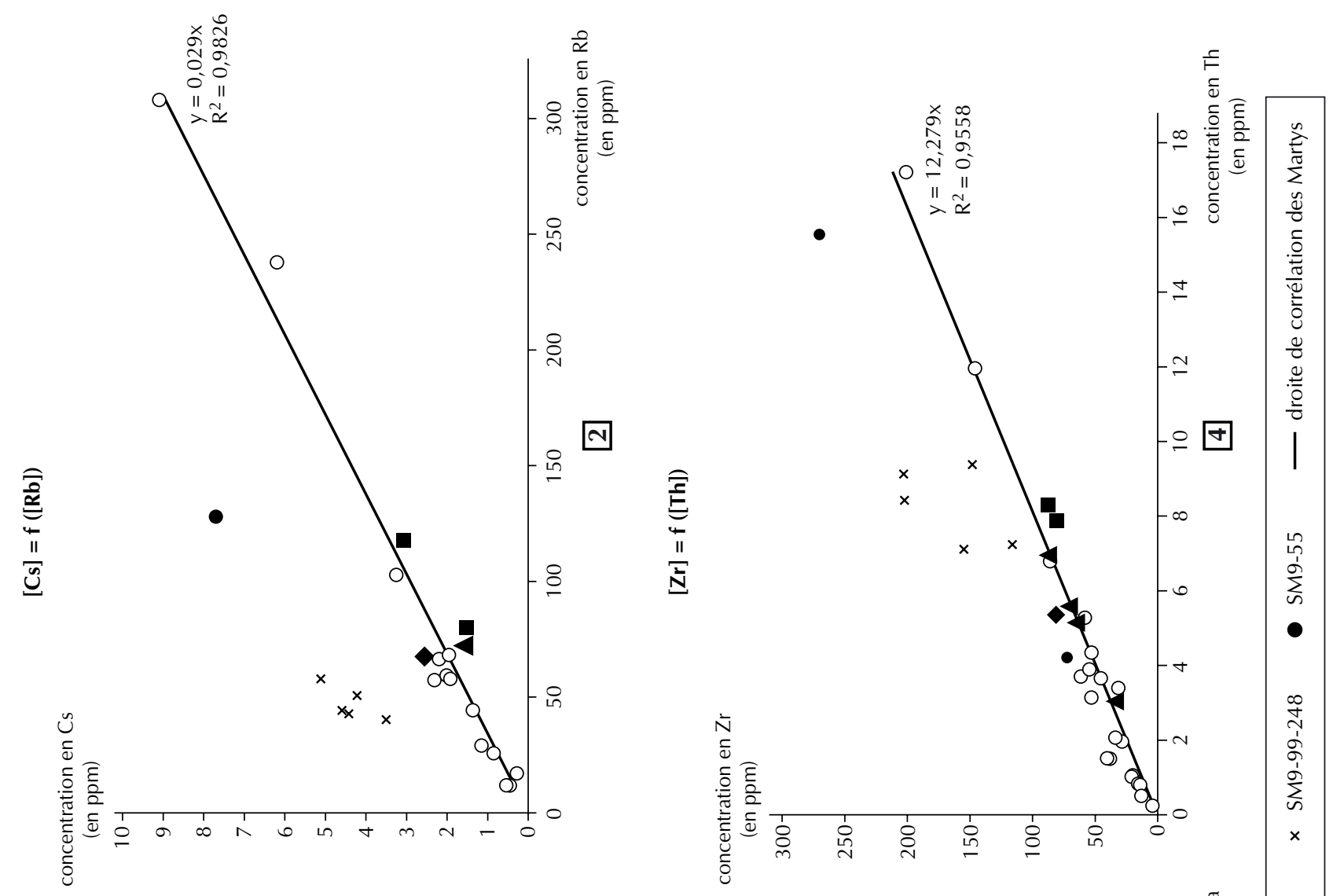

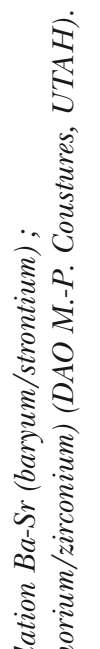
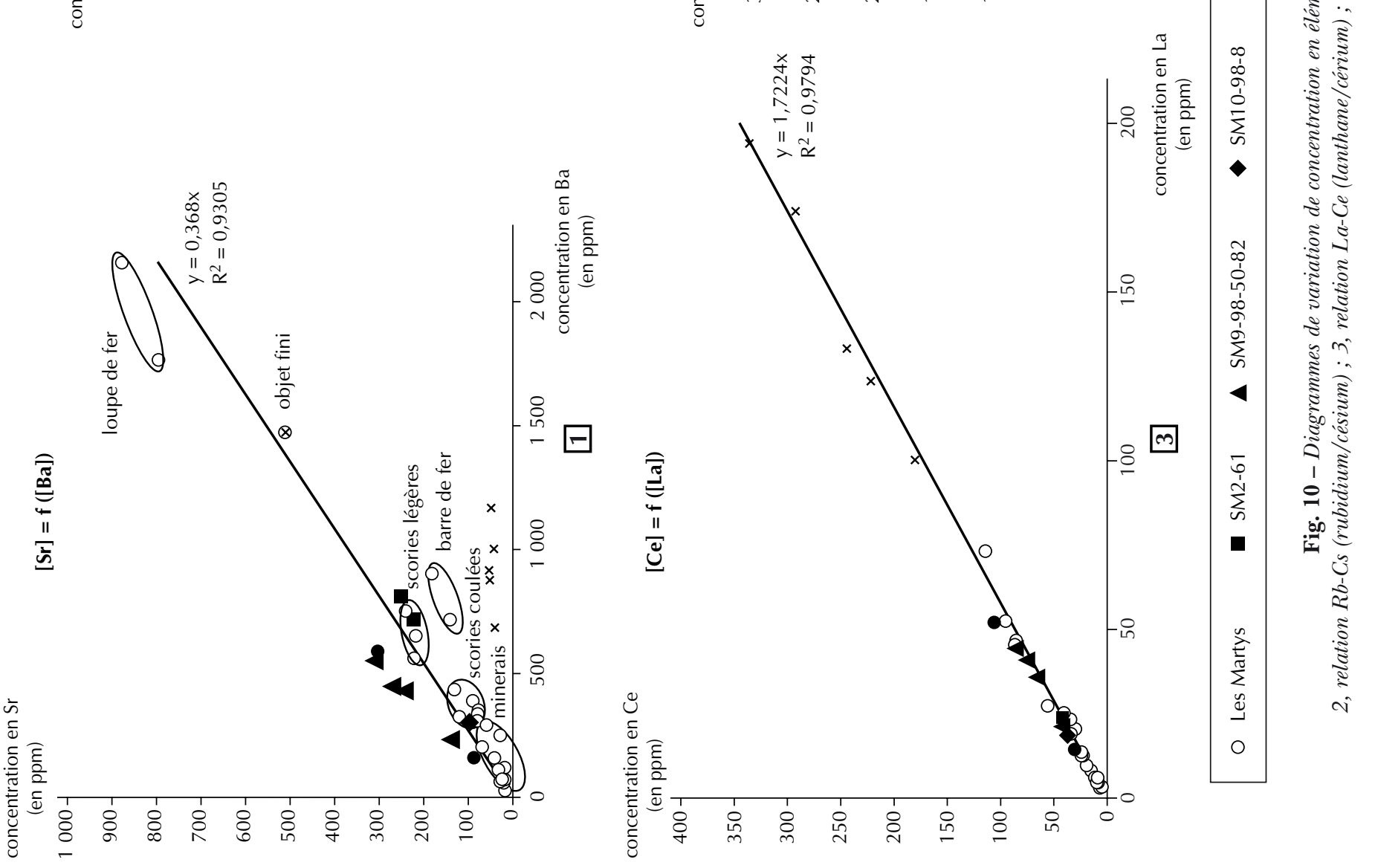


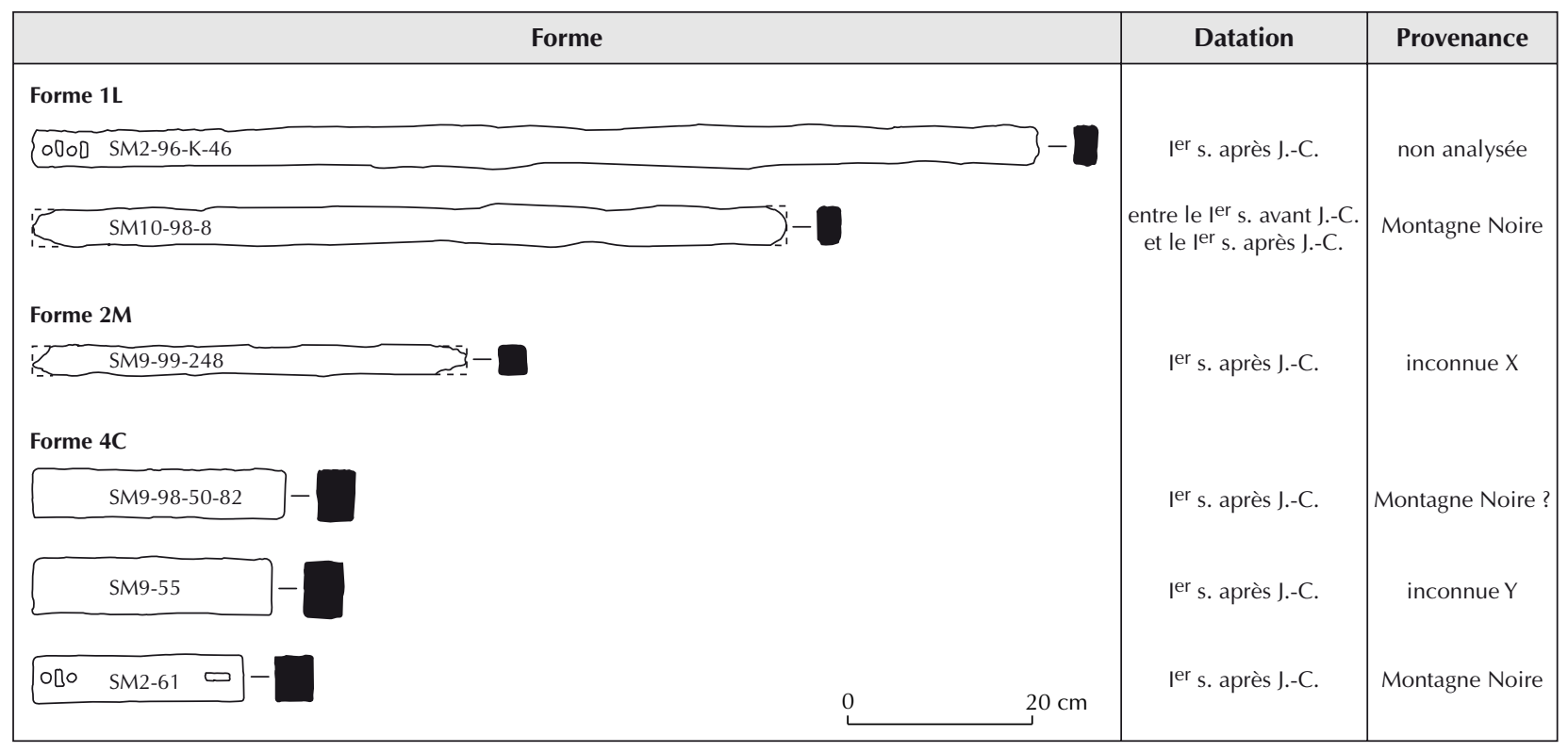

Fig. 11 - Synthèse des résultats des analyses (DAO M.-P. Coustures, UTAH).

épaves des Saintes-Maries-de-la-Mer, mais tend plutôt à mettre en exergue les divers enseignements d'une telle étude, toute préliminaire soit-elle.

Les barres de fer SM2-61 et SM10-98-8 présentent, en tous points, la même signature chimique que le centre sidérurgique des Martys. La signature chimique de la barre de fer SM 9-98-50-82 ne diffère de celle des Martys que pour un seul couple d'éléments en trace (Ba-Sr), dont la teneur a pu être fortement influencée par la composition du revêtement argileux du bas fourneau. Compte tenu de ces similitudes chimiques et des arguments archéologiques, cités supra, qui font de la Montagne Noire, et du centre sidérurgique des Martys en particulier, le secteur de provenance le plus probable pour les barres de fer des Saintes-Mariesde-la-Mer, on peut valider l'hypothèse selon laquelle les barres de fer SM2-61, SM10-98-8 et peut-être SM9-98-50-82 ont été produites aux Martys.

La signature chimique d'un centre sidérurgique dépendant du minerai utilisé pour la réduction, il sera donc impossible de différencier chimiquement deux lieux de production différents ayant utilisé le même minerai. Le centre des Martys n'étant pas le seul à avoir fonctionné dans la Montagne Noire à l'époque de la production des barres de fer des Saintes-Maries-de-la-Mer (Decombeix et al., 2000, p. 32-33), d'autres centres sidérurgiques sont susceptibles d'avoir utilisé le même minerai qu'aux Martys et, par conséquent, de présenter la même signature chimique.
La provenance des barres SM2-61, SM10-98-8 et peut-être SM9-98-50-82 devrait ainsi être plus justement attribuée à la Montagne Noire en général qu'au centre sidérurgique des Martys proprement dit.

La différence quasi systématique entre la signature chimique des barres de fer SM9-99-248, SM9-55 et celle des Martys laisse penser que ces deux barres n'ont pas été produites dans la Montagne Noire. Leur provenance demeure donc pour l'instant inconnue. De plus, les signatures chimiques de ces deux barres n'étant pas semblables, elles peuvent provenir de deux centres sidérurgiques différents.

La barre SM9-99-248, qui se distinguait déjà par une proportion très importante de scories en inclusion dans le fer, est ainsi chimiquement différente des autres barres des Saintes-Maries-de-la-Mer. Les différences de qualité du fer, plus ou moins épuré, qui pourraient témoigner de savoirfaire distincts, peuvent donc être mises en relation avec la diversité des lieux de production. Ce critère discriminant supplémentaire devra désormais être pris en compte dans la recherche des origines des barres de fer des épaves des Saintes-Maries-de-la-Mer.

Ainsi, les analyses des échantillons, tels qu'ils avaient été choisis, ont permis non seulement de tester l'hypothèse de provenance du centre sidérurgique des Martys, mais aussi de montrer que d'autres provenances étaient possibles, ce qui révèle toute la complexité de ce commerce du fer au $\mathrm{I}^{\mathrm{er}}$ s. av. et au I $\mathrm{I}^{\mathrm{er}}$ s. apr. J.-C. 
On savait depuis longtemps que la Montagne Noire avait constitué à l'époque romaine un important complexe sidérurgique, comme le prouvent le nombre et la dispersion des ferriers qui y ont été recensés et dont certains témoignent par leur volume d'une grande activité passée (Domergue dir., 1993, p. 16-18; Decombeix et al., 2000, p. 33), mais on ignorait ce que devenait le métal qui y était produit. Il n'en va plus de même aujourd'hui, après la découverte des épaves chargées de fer des Saintes-Maries-de-la-Mer et après les analyses qui ont été pratiquées sur des barres de commerce prélevées dans ces cargaisons. On a ainsi montré que:

- dans un même bateau (SM9) se trouvaient des barres d'au moins trois origines différentes, dont une provenait peutêtre de la Montagne Noire (fig. 11) ;

- dans trois épaves différentes (SM2, SM10 et peut-être $S M 9)$, il y avait des barres de fer qui ont pu être fabriquées dans un des centres sidérurgiques de la Montagne Noire;

- un même centre sidérurgique, celui des Martys, a produit des barres de fer, estampillées ou non, et de formes différentes.

Mais certaines barres semblent aussi provenir d'autres régions (fig. 1). Dans la mesure où, selon toute probabilité, Narbonne a été un des ports de chargement des bateaux, on peut penser à des régions proches, les Corbières peutêtre, mais surtout le Canigou, dont la production de fer est attestée au I ${ }^{\text {er }}$ s. av. et au I ${ }^{\mathrm{er}}$ s. apr. J.-C. Il faudra donc étendre de ce côté au moins l'échantillonnage et les analyses.

Narbonne, cependant, a pu aussi être un port de redistribution de cargaisons de fer venues d'autres régions par voie maritime. Dans cette perspective, on ne peut refuser l'hypothèse de provenances plus lointaines, au moins théoriquement, puisque, pour l'instant, les informations sont assez rares sur des secteurs de la péninsule Ibérique comme la Sierra Menera (Teruel) ou la Sierra Nevada (Grenade), voire la Sierra de la Demanda (Burgos, Logroño), qui ont pu être actives à cette période.

Dans ce domaine de la production, les estampilles doivent être prises en considération. L'étude onomastique semble, elle aussi, orienter vers Narbonne et sa région. Mais il faut encore tenir compte de la façon dont les barres sont marquées: association, organisation et position des timbres.
La texture du marquage est uniforme et peu variée: des timbres rectangulaires allongés - dont la plupart sont inscrits, mais l'un d'eux porte un symbole, une ancre marine - et des timbres plus petits, de forme circulaire. Ces timbres peuvent être isolés (un seul par barre), mais sont toujours soigneusement et régulièrement imprimés; ils sont surtout fréquemment associés selon des agencements assez divers, mais qui présentent quelque ressemblance (tabl. II). Ils occupent des emplacements bien précis sur les barres, soit au milieu, soit à l'une des extrémités. Ces remarques tendraient plutôt à circonscrire une aire de production de ce fer, assez bien délimitée et caractérisée par ce genre de marquage spécifique, dont ferait obligatoirement partie la Montagne Noire. Mais jusqu'où cette aire pourrait-elle s'étendre? Jusqu'aux Corbières et aux Pyrénées? Ou plus loin encore? Des campagnes d'analyses à venir devraient permettre de le préciser.

La composition des cargaisons constitue un autre problème. Prenons encore l'exemple de Narbonne: ce port est certes le débouché naturel des productions de la Montagne Noire, mais les docks des bassins d'embarquement ont pu également abriter des stocks de barres provenant de centres sidérurgiques de différentes régions. On ne saurait donc trop s'étonner de trouver dans une même cargaison des barres d'origines diverses, comme le montrent les analyses. On pourrait tout aussi normalement avoir affaire à des cargaisons homogènes, qui contiendraient des barres provenant d'un seul centre. Cela devait dépendre des arrivages à Narbonne, de la façon dont ce fer était stocké (plusieurs hangars ou non, dispersés ou non, etc.), des pratiques commerciales et de la manière dont ces stocks étaient gérés, par des marchands et/ou par une autorité supérieure (Domergue et al., 2003). Dans ce domaine aussi, l'archéométrie devrait permettre d'aller plus loin.

\section{Nota bene}

Nous remercions F. Dabosi (CIRIMAT, LIMAT, UMR $5085 \mathrm{du}$ CNRS, Institut national polytechnique de Toulouse) d'avoir bien voulu relire le paragraphe concernant l'étude métallographique du métal des barres. 


\section{BIBLIOGRAPHIE}

ANDRIEUX P., JARRIER C., DOMERGUE C., Pieraggi B., Ploquin A., Tollon F.

1997 : "Élaboration du fer par réduction directe: essais de reproduction des procédés antiques ", La Revue de métallurgie-CIT/Science et génie des matériaux, p. 691-704.

\section{BARRouillet V., LAMY A., MUT G.,} Pinéda J., SiRet A.

1991 : «Mines et fonderies antiques et médiévales du Canigou ", in Domergue C. (DIR.), Mines et métallurgies antiques et médiévales de la France méridionale. Recherches récentes, Journées de Perpignan, févr. 1987, Perpignan, Ville de Perpignan, p. 21-29.

\section{BÉMONT C.}

1991: «Le statut des patrons et des ouvriers d'après les marques de fabriques ", Specimina Nova Universitatis Quinqueecclesiensis, p. 133-156.

\section{COUSTURES M.-P.}

2001: D'où proviennent les barres de fer des épaves antiques des Saintes-Maries-de-la-Mer (Bouches-du-Rhône)?, mémoire de DESS (multigraphié), Dijon, université de Bourgogne.

Coustures M.-P., Béziat D., Tollon F., DOMERGUE C., LONG L., REBISCOUL A.

2003: "The use of trace element analysis of entrapped slag inclusions to establish ore - bar iron links: examples from two Gallo-Roman iron-making sites in France (Les Martys, Montagne Noire and Les Ferrys, Loiret)", Archaeometry, 45,4 , p. 589-603.

CREW P.

2000 : "The influence of clay and charcoal ash on bloomery slags ", in CUCINITIZZONI C., TIZZONI M. (EDS), LeFerdans les Alpes/Iron in the Alps, Actes du colloque international de Bienno (Italie), oct. 1998 , Breno, comune di Bienno, p. 38-48.

DECOMBEIX P.-M., DOMERGUE C., FABRE J.-M., GORgUES A., RICO C., TOLLON F., TOURNIER B.

2000: «Réflexions sur l'organisation de la production de fer à l'époque romaine dans le bassin supérieur de la Dure, au voisinage des Martys (Aude) ", in Domergue C., LEROY M. (DIR.), "Dossier: Mines et métallurgies en Gaule », Gallia, 57, p. 23-36.
DECOMBEIX P.-M., DOMERGUe C., FABRE J.-M., TOLLON F.

1998: «Évaluation du volume des ferriers romains $\mathrm{du}$ domaine des Forges (Les Martys-Aude), de la masse de scories qu'ils renferment et de la production de fer correspondante ", Revue d'Archéométrie, 22, p. 77-90.

\section{DillmanN P.}

1998 : Diffraction X, microdiffraction X et microfluorescence $X$ sous rayonnement synchrotron et analyses comparées pour la caractérisation des inclusions. Application à létude de l'évolution historique des procédés d'élaboration des objets ferreux, thèse de Doctorat, Compiègne, université de Technologie, 2 vol.

\section{DILLMANN P., BALASUBRAMANIAM R.}

2001 : " Characterization of ancient Indian iron and entrapped slag inclusions using electron, photon and nuclear microprobes ", Bulletin of Materials Science, 24, 3, p. 317-322.

\section{DJAOUI D.}

2003: Le Commerce du fer d'après les épaves des Saintes-Maries-de-la-Mer $(50$ av. J.-C.90 apr. J.-C.), mémoire de DEA (multigraphié), Aix-en-Provence, université de Provence.

\section{DOMERGUE C. (DIR.)}

1993: Un Centre sidérurgique romain de la Montagne Noire. Le domaine des Forges (Les Martys, Aude), Paris, CNRS Éditions (coll. Suppl. à la Revue archéologique de Narbonnaise, 27), $477 \mathrm{p}$

DOMERgue C., BENQUET L., DECOMBEIX P.-M., FABRE J.-M., GORGUES A., Rico G., TOLlON F.

2003 : « La Guerre des Gaules et les débuts de la sidérurgie romaine en Montagne Noire ", Pallas, 63, p. 241-247.

\section{DOMERGUE C., LIOU B.}

1997: «L'apparition de normes dans le commerce maritime romain : le cas des métaux et des denrées transportées en amphores ", in Mélanges Claude Domergue, 1, Pallas, 46, p. 11-30.

FEUGÈre M., SERNEELS V.

1996 : « Production, commerce et utilisation du fer entre L'Èbre et le Rhône : premiers éléments de réflexion », in FEUGÈRE M.,
SERNEELS V. (DIR.), Recherches sur l'économie du fer en Méditerranée nordoccidentale, Montagnac, Monique Mergoil (coll. Monographies Instrumentum, 4), p. $251-263$.

\section{Hedges R. E. M., SAlter C. J.}

1979: "Source determination of iron currency bars through analysis of the slag inclusions ", Archaeometry, 21, 2, p. $161-175$.

\section{JARRIER C.}

1993: Minéralogie, pétrologie et géochimie des résidus sidérurgiques gallo-romains (Ariège et Montagne Noire). Comparaison avec les résultats des essais in situ de réduction directe $d u$ fer, thèse de Doctorat, Toulouse, université Paul-Sabatier, 2 vol. (multigraphiés) : 225 p. et $170 \mathrm{p}$.

JARRIER C., DOMERGUe C., Pieraggi B., PloQuin A., Tollon F.

1995 : «Caractérisation minéralogique, géochimique et métallurgique des résidus de réduction directe, d'épuration et de forge du centre sidérurgique romain des Martys (Aude, France) ", Revue d'Archéométrie, 19, p. 49-61.

1996 : «Archéologie et archéométrie de la sidérurgie romaine dans la Montagne Noire au I ${ }^{\mathrm{er}}$ siècle avant J.-C. Le cas des Martys (Aude)", Bulletin de la Société des études scientifiques de l'Aude, 96, p. 11-22.

\section{LEBLANC J.-C.}

2002 : Sur l'identification de la chaîne opératoire de forgeage du fer antique, associée à l'archéométrie des battitures, thèse de Doctorat, Toulouse, université Paul-Sabatier, 2 vol. (multigraphiés) : 189 p. et 48 p.

\section{LONG L.}

1985 : L'Épave antique Bagaud 2, VI Congreso international de Arqueología submarina, Cartagena, 1982, Madrid, p. 93-98.

1995 : "Bouches-du-Rhône. Au large des Saintes-Maries-de-la-Mer ", Bilan scientifique du Département des recherches archéologiques subaquatiques et sous-marines, Paris, ministère de la Culture et de la Communication, p. 40-44.

1997 : "Inventaire des épaves de Camargue, de l'Espiguette au Grand Rhône, Des cargaisons de fer antiques aux gisements du $\mathrm{XIX}^{\mathrm{e}}$ siècle. Leur contribution à l'étude du paléorivage ", in Actes du colloque Crau, Alpilles, Camargue, histoire et archéo- 
logie, Groupe archéologique arlésien, p. 59-115.

1998: "Carte archéologique. Au large de la Camargue ", Bilan scientifique du Département des recherches archéologiques subaquatiques et sous-marines, Paris, ministère de la Culture et de la Communication, p. 32-37.

2002: «Épave Saintes-Maries-de-la-Mer 24 », Bilan scientifique du Département des recherches archéologiques subaquatiques et sousmarines, Paris, ministère de la Culture et de la Communication, p. 53-57.

LONG L., DOMERGUE C.

1995 : "Le "véritable plomb de L. Flavius Verucla” et autres lingots. L'épave 1 des Saintes-Maries-de-la-Mer », Mélange de l'École française de Rome, Antiquité, 107, 2, p. 801-867.

LONG L., RICO C., DOMERGUE C.

2002: "Les épaves antiques de Camargue et le commerce maritime du fer en Méditerranée nord-occidentale $\left(\mathrm{I}^{\mathrm{er}}\right.$ siècle avant J.-C.-- ${ }^{\mathrm{er}}$ siècle après J.-C.) ", in KHANOUSSI M., RUGGERI P., VIZMARA C. (DIR.), L'Africa romana. Lo Spazio marittimo del Mediterraneo occidentale : geografia storica ed economia, Atti del XIV convenio di studi, Sassari, 7-10 dic. 2000, Rome, Carocci Editore, p. 161-188.

LONG L., SINTÈS C.

2003: «Commerce maritime et fluvial aux embouchures du Rhône: le rôle d'Arles dans l'Antiquité ", in PASCUAL Berlauga G., PÉrez Ballester J. (DIR.), Puertos fluviales antiguos: ciudad, desarollo e infraestructuras, Actas de las IV Jornadas de arqueología subaquática, Valencía, 28-30 de març de 2001, Valence, Université de Valence, p. 183-201.

\section{LONG L., VALENTE M.}

2003: «Un aspect des échanges et de la navigation depuis l'Antiquité dans les îles et sur le littoral d'Hyères. Synthèse des récents travaux du DRASSM ", in PASQUALINI M., ARNAUd P., VARALDO C. (DIR.), Des Îles côte à côte. Histoire du peuplement des îles de l'Antiquité au Moyen Âge (Provence, Alpes-Maritimes, Ligurie, Toscane), Actes de la table ronde de Bordighera, 12-13 déc. 1997, Aix-en-Provence-Bordighera (coll. Suppl. au Bulletin archéologique de Provence, 1), p. 149-164.

\section{MAHÉ-LE CARLIER C.}

1997 : Caractérisation pétrographique et chimique d'analogues de déchets vitrifiés actuels: les scories de la métallurgie ancienne. Étude de l'altération naturelle et expérimentale, thèse de Doctorat (multigraphiée), Institu national polytechnique de Lorraine, $395 \mathrm{p}$.

\section{MANGIN M. (DIR.)}

2004: Le Fer, Paris, éd. Errance (coll. Archéologiques), 239 p.

\section{Pauc B., Pauc P.}

1998: «Quelques sites de réduction du minerai de fer dans les Corbières (Aude) », in FEUgÈRe M., SERnEELS V. (DIR.), Recherches sur l'économie du fer en Méditerranée nord-occidentale, Montagnac, Monique Mergoil (coll. Monographies Instrumentum, 4), p. 116-128.

\section{Ploquin A.}

2004a: "Prélèvement, échantillonnage et représentativité", in MANGIN M. (DIR.), Le Fer, Paris, éd. Errance (coll. Archéologiques), p. 117-118.

2004b: "Préparation des spécimens ", in MANGIN M. (DIR.), Le Fer, Paris, éd. Errance (coll. Archéologiques), p. $124-125$.

\section{RANCOULE G.}

1975 : «Une petite exploitation minière dans les Corbières: Montjoi (Aude)", Actes du $98^{e}$ congrès national des Sociétés savantes
(Saint-Étienne, 1973), section d'archéologie et d'histoire de l'art, archéologie minière, Forez et Massif central, Paris, p. 93-100.

RENOUX G., PAILler J.-M., DABosi F.

2001 : «Première étude paléométallurgique des armes de fer du Puy d'Issolud (Lot) ", La Revue de métallurgie-CIT/Science et génie des matériaux, p. 1147-1158.

\section{ROTHENHÖFER P.}

2003a: "Geschäfte in Germanien. Zur Ausbeutung von Erzlagerstätten unter Augustus in Germanien ", Zeitschrift für Papyrologie und Epigraphik, 143, p. 277-286.

2003b: "Plumbum Germanicum. Germanic lead and the history of Augustean Germany ", in Archaeometallurgy in Europe, international conference, 24-26 sept. 2003, Milan, Proceedings, Milan, Associazione italiana di metalurgia, vol. 2, p. 641-648.

\section{SERDON F., FLUZIN P.}

2002: «Étude paléométallurgique des fers de traits du Moyen Âge, contribution à l'histoire des techniques ", Revue d'Archéométrie, 26, p. 209-218.

SOLIER Y. (DIR.)

1981 : "Les épaves de Gruissan ", Archaeonautica, 3, p. 7-264.

\section{TRINCHERINi P. R., BARbERo P.,} QUARATI P., DOMERGUE C., LONG L.

2001: "Where do the lead ingots of the Saintes-Maries-de-la-Mer wreck come from? Archaelogy compared with physics ", Archaeometry, 43, p. 393-406.

\section{ZWICKER U.}

1996 : «Ein Werkzeugstahl des Saturninus », Bayerisches Vorgeschichtsblatt, 61, p. $245-246$ 